\title{
Tradeoffs and Theory: The Double-Mediation Model
}

\author{
Marc Scholten \\ Instituto Superior de Psicologia Aplicada
}

\author{
Steven J. Sherman \\ Indiana University
}

\begin{abstract}
Most theories of decision making suggest that, when options imply tradeoffs between their attributes, conflict increases as tradeoff size increases, because greater sacrifices are to be incurred in choosing one option instead of another. An alternative view is that conflict decreases as tradeoff size increases, because stronger arguments can be made for any decision. The authors propose a unified model, the doublemediation model, which combines the mediating effects of sacrifice and argumentation. Our model generally predicts an inverse $U$-shaped relation between tradeoff size and conflict. Results support this prediction. Also, when the decision situation increases the mediating effect of sacrifice relative to that of argumentation, the relation between tradeoff size and conflict changes in an upward direction; conversely, when the decision situation increases the mediating effect of argumentation relative to that of sacrifice, the relation changes in a downward direction. Results support these predictions as well. Commonalities and differences between our model and other formulations are discussed.
\end{abstract}

Keywords: argumentation, conflict aversion, orientation reaction, vacillation, sacrifice

Most theories of decision making suggest that conflict arises when the options under consideration imply tradeoffs between their attributes, that it increases with the size of the tradeoffs, and that it decreases with the difference in importance between the attributes (Chatterjee \& Heath, 1996; Scholten, 2002). Regarding tradeoff size, conflict is believed to be greater when more or larger advantages of one option are to be traded off against more or larger advantages of another option (Festinger, 1964). However, when asked about this, people often express the opposite belief, that conflict is greater when the advantages to be traded off are few and small than when they are many or large. When asked why this would be so, they typically answer that, when the advantages to be traded off are few and small, any decision is tenuous, in that it is difficult to find good reasons for choosing any option. Thus, a common intuition, which has its appeal from a reason-based perspective on decision making (Shafir, Simonson, \& Tversky, 1993; Simonson, 1989), is that conflict decreases, rather than increases, with tradeoff size.

In this article we discuss a unified model, the double-mediation model, which suggests that conflict may both increase with

Marc Scholten, Instituto Superior de Psicologia Aplicada, Lisbon, Portugal, Steven J. Sherman, Indiana University, Bloomington.

We are grateful for the financial support received from the Fundação para a Ciência e Tecnologia (FCT), project POCTI/PSI/42275/2001. The data were collected with the invaluable assistance of Luís Brito and Tânia Correia (Experiment 1); Estela Baptista, Inês Rosa, and José Silva (Experiment 2); and Alexandre Fernandes (throughout). The moderator variable of Experiment 2 (need for justification) was suggested to us by Itamar Simonson. The article has greatly benefited from discussions with John Cacioppo, Manuela Faia Correia, Daniel Read, Inês Rosa, and Sandra Schneider and from comments on earlier drafts by Jerome Busemeyer, Manuela Faia Correia, Edward Hirt, Daniel Read, Inês Rosa, and James Townsend.

Correspondence concerning this article should be addressed to Marc Scholten, Instituto Superior de Psicologia Aplicada, Rua Jardim do Tabaco 34, 1149-041 Lisbon, Portugal. E-mail: scholten@ispa.pt tradeoff size, because larger tradeoffs mean that greater sacrifices are to be incurred in choosing one option instead of another, and decrease with tradeoff size, because larger tradeoffs mean that stronger arguments can be made for any decision. We derive testable implications from this model, which were tested in two experiments.

\section{Defining Conflict}

When faced with tradeoffs, people are often not sure which decision to make and not sure whether they would make the same decision on a different occasion (Tversky \& Sattath, 1979). Although conflict has no formal definition (Tversky \& Shafir, 1992), it is usually described as such a state of preference uncertainty, which suffices as an informal definition.

\section{Predicting Conflict}

In our experiments, participants were presented with several decision domains, mostly consumer products with which they were familiar and with which they had affinity. On each occasion, they were presented with a particular product, for example, cellular phones, and with two options that differ along two continuous attributes, for example, talk time and weight. The options implied a tradeoff between those attributes, in that one option was better along one attribute, for example, more talk time, but the other option was better along the other attribute, for example, less weight, and the participants were asked to choose. We examined how conflict is related to the size of the tradeoff and how this relation is moderated by other aspects of the decision situation. In Experiment 1, we focused on an aspect that is ubiquitous in decision making: the difference in importance between the attributes under consideration.

A model of conflict generation necessarily draws on assumptions about preference formation. For instance, if it is assumed that preferences are formed simply by determining which option is better along the more important attribute, without any consideration of the tradeoff between the attributes (i.e., lexicographic 
ordering; see Fishburn, 1974), then conflict should be unrelated to tradeoff size. Our model, however, suggests that conflict $i$ s related to tradeoff size, that this relation can take on many forms, and that the specific shape depends on the decision situation.

\section{Assumptions About Preference Formation}

The double-mediation model derives its assumptions about preference formation from (multiattribute) decision field theory (Busemeyer \& Diederich, 2002; Busemeyer \& Townsend, 1993; Diederich, 1997). It assumes that preferences are formed by a preliminary impression of the decision problem and subsequent deliberation about the decision problem. When a decision maker is presented with a decision problem, a preliminary impression is formed on the basis of prior knowledge about or past experience with the decision domain. The preliminary impression is translated into an initial preference state, which may be neutral or not. For instance, if, on the basis of prior knowledge about or past experience with cellular phones, talk time is more important to the decision maker than weight, the initial preference state will favor the option that has a longer talk time. Generally, however, the decision maker will not simply choose that option right there and then but will start deliberating about which option to choose.

Deliberation is a process of considering and reconsidering attributes, comparing the options along the attribute that is being considered at a particular moment, contemplating consequences of choosing one option instead of the other, evaluating the options on the basis of those consequences, and updating the preference state by the value difference between the options. For instance, when the weight of the cellular phones is being considered, the preference state will be adjusted in favor of the option that has less weight, but the magnitude of the adjustment will depend on the consequences that are being contemplated at that moment. Deliberation stops when the preference for one of the options is, according to a criterion set by the decision maker, strong enough. That option is then chosen.

The above process of preference formation (or, rather, preference evolution) is formally described by decision field theory. This stochastic-dynamic theory of decision making predicts the probability that a particular decision will be made and the average time it will take to make that decision. If decision time is viewed as a manifestation of conflict, as it has been in applications of conflict theory (Berlyne, 1957a; but see Berlyne, 1962, 1966) and decision field theory (Diederich, 2003), then decision field theory can be viewed as a theory of decision making and a theory of the conflict involved in decision making. However, it has thus far not been applied to the question how conflict is related to the size of the tradeoff between the attributes under consideration and how this relation is moderated by other aspects of the decision situation, for example, the difference in importance between the attributes. Moreover, the double-mediation model, in addressing this question, identifies two sources of conflict in preference formation, namely, concern about the sacrifices that are to be incurred in choosing one option instead of the other as well as concern about the arguments that can be made in favor of either decision: It is not obvious how these sources of conflict are to be defined within the formal machinery of decision field theory. Therefore, we develop the double-mediation model as an autonomous model of conflict generation, drawing on the assumptions about preference formation from decision field theory.
The double-mediation model invokes two sets of assumptions about conflict generation: (a) those motivated by the process of preference formation and (b) those motivated by the operation of two sources of conflict in that process. To show more clearly the implications of each set of assumptions and to smooth the transition from the state of the art to the double-mediation model, we first discuss a single-mediation model, which suggests that concern about sacrifice is the only source of conflict in the process of preference formation. This sets the stage for the development of the double-mediation model, which suggests that concern about sacrifice and concern about argumentation are both sources of conflict in that process.

\section{Assumptions of the Single-Mediation Model}

As mentioned earlier, most theories of decision making suggest that conflict increases with tradeoff size. One rationale for a positive relation between tradeoff size and conflict is provided by the theory of cognitive dissonance. According to Festinger (1964), conflict before a decision has the same origin as cognitive dissonance after the decision: the things that the decision maker knows are inconsistent with a particular decision. The greater the number of inconsistencies, the greater the conflict and subsequent cognitive dissonance. For instance, the choice between cellular phones $X=$ (longer talk time, larger memory store) and $Y=$ (longer standby time, less weight), where each option has two advantages and two disadvantages, should arouse more conflict than the choice between $x=$ (longer talk time) and $y=$ (less weight), where each option has only one advantage and one disadvantage. What applies to the number of inconsistencies also applies to the magnitude of the inconsistencies (see Shugan, 1980). For instance, the choice between $x_{L}=(5 \mathrm{hr}, 160 \mathrm{~g})$ and $y=(2 \mathrm{hr}, 70 \mathrm{~g})$, where the options differ by $3 \mathrm{hr}$ in talk time and $90 \mathrm{~g}$ in weight, should arouse more conflict than the choice between $x_{S}=\left(2 \frac{1}{2} \mathrm{hr}, 85 g\right)$ and $y=(2 \mathrm{hr}, 70 \mathrm{~g})$, where the options differ by only $1 / 2 \mathrm{hr}$ in talk time and $15 \mathrm{~g}$ in weight.

Another rationale for a positive relation between tradeoff size and conflict is provided by prospect theory. According to Kahneman and Tversky (1979), losses are more painful than equivalent gains are pleasant and, according to Simonson and Tversky (1992; Tversky \& Simonson, 1993), this also applies to relative gains and losses: Disadvantages are more painful than equivalent advantages are pleasant. Thus, larger tradeoffs lead to a greater unattractiveness of both options under consideration, which amplifies the lose-lose nature of the decision and intensifies the vacillation between the options (Chatterjee \& Heath, 1996). In general, larger tradeoffs mean that greater sacrifices are to be incurred in choosing one option instead of the other, leading to a greater conflict. This proposal is incorporated into the single-mediation model, which is developed below in three steps.

Updating conflict. The single-mediation model identifies concern about sacrifice as the only source of conflict in preference formation. Conflict is elicited from this source by a preliminary impression of the decision problem and by subsequent deliberation about the decision problem. The conflict from the preliminary impression is updated by the conflict from deliberation into final conflict. Obviously, the more the conflict from the preliminary impression is updated by the conflict from deliberation, the less the former contributes to final conflict. 
Relation with tradeoff size. Preference formation is described as a process in which a preliminary impression is formed on the basis of prior knowledge about or past experience with the decision domain and in which, during subsequent deliberation, the options are compared along their attributes and the consequences of choosing one option instead of the other are contemplated. Thus, it is only during deliberation that the tradeoff between the attributes is considered and that conflict is affected by the size of the tradeoff. According to the single-mediation model, conflict is positively affected by tradeoff size, because larger tradeoffs mean greater sacrifices, leading to a greater conflict.

Drive for deliberation. As preference formation is described, choice is generally not dictated by the preliminary impression but by subsequent deliberation. The question arises, Why would people even bother deliberating? An obvious answer, in the spirit of decision field theory, is that they are not oriented toward a choice that is trouble-free but rather toward a choice that is good enough. However, this answer begs the question, What is the origin of that orientation?

Although conflict is generally believed to have a paralyzing effect on an individual by producing vacillation between alternative courses of action, Berlyne (1961) has shown that it also has a mobilizing effect on the individual by producing a drive to gather information about the alternative courses of action and to get ready for action, that is, an orientation reaction: The greater the conflict, the greater the drive (see also the evidence reviewed by Berlyne, 1957b, and the evidence reported by Bettman, Johnson, Luce, \& Payne, 1993). Thus, preference formation can be viewed as a process in which conflict first has a mobilizing effect (getting ready) and only then a paralyzing effect (getting stuck). If you don't get ready, you won't get stuck. Consistent with this proposal, the single-mediation model suggests that the conflict from the preliminary impression is the drive for deliberation: The greater the conflict, the greater the drive.

In sum, the single-mediation model assumes that the conflict from the preliminary impression is the drive for deliberation, that the conflict from deliberation increases with tradeoff size, and that the conflict from the preliminary impression is updated by the conflict from deliberation into final conflict. A formal statement of these assumptions follows below.

\section{Formulation of the Single-Mediation Model}

Applying Anderson's (1971) integration theory, the updating of conflict can be captured by a weighted averaging model:

$$
C=\left(1-w_{S}\right) S_{0}+w_{S} S_{1},
$$

where $S_{0}, S_{1} \geq 0$ and $0 \leq w_{S}<1$. In this formulation, $w_{S}$ is an updating parameter, which covers everything from no updating at all, when $w_{S}=0$, to complete updating, as $w_{S} \rightarrow 1$. In addition, $S_{0}$ is the conflict from the preliminary impression, $\left(1-w_{S}\right) S_{0}$ is the residual conflict from the preliminary impression after the updating, $w_{S} S_{1}$ is the conflict from deliberation, and $C$ is final conflict. A weighted averaging model is equivalent to an anchoring-and-adjustment model:

$$
C=S_{0}+w_{S}\left(S_{1}-S_{0}\right),
$$

which describes an anchoring on the conflict from the preliminary impression, $S_{0}$, and an adjustment by the conflict from delibera- tion, $w_{S}\left(\mathrm{~S}_{1}-S_{0}\right)$. Given this formulation, the single-mediation model invokes two additional assumptions, one about $S_{1}$ and the other about $w_{S}$. First, to capture the positive relation between tradeoff size and conflict, $S_{1}$ is defined as a function of tradeoff size, $T$, which itself is a function of the perceived difference between the two options along each of the two continuous attributes (see our experiments):

$$
S_{1}=\mathbf{f}_{S}(T),
$$

where $T \geq 0$ and $\mathbf{f}_{S}$ is an increasing function. For simplicity, we assume in this article that $\mathbf{f}_{S}$ is a linear function. Second, to capture the capacity of the preliminary conflict as a drive for deliberation, $w_{S}$ is defined as a function of $S_{0}$ which itself is a function of other aspects of the decision situation, for example, differential attribute importance (see below):

$$
w_{S}=\mathbf{g}\left(S_{0}\right),
$$

where $g$ is an increasing function. In terms of Anderson's (1971) integration theory, Equation 3 changes Equation 1 into a differentially weighted averaging model, in that the relative weight of the preliminary conflict, $1-w_{S}$, is inversely related to its level, $S_{0}$ : The conflict from the preliminary impression curtails its own contribution to final conflict by raising the drive for deliberation. Below, we discuss the implications of the single-mediation model for the relation between tradeoff size and conflict.

\section{Implications of the Single-Mediation Model}

The single-mediation model predicts that conflict will generally have a positive relation with tradeoff size but that this relation will be moderated by other aspects of the decision situation, for example, differential attribute importance. Imagine a person who, on the basis of prior knowledge about or past experience with cellular phones, believes that talk time is about as important as weight, a second person who believes that talk time is more important than weight, and a third person who believes that talk time is much more important than weight. ${ }^{1}$ The single-mediation model predicts that differential attribute importance will decrease the conflict from the preliminary impression (see the decreasing flat lines denoted $S_{0}$ in Figure 1A), because incurring a sacrifice along the less important attribute seems less painful than incurring a sacrifice along the more important attribute. Moreover, the singlemediation model predicts that a decreasing conflict from the preliminary impression will attenuate the relation between tradeoff size and final conflict (see the flattening lines denoted $C$ ), because it decreases the drive for deliberation.

We emphasize that the moderating effect of situational aspects (in the above example, the attenuating effect of differential attribute importance) follows only from the differentially weighted averaging model, in which the updating parameter, $w_{S}$, varies with the preliminary conflict, $S_{0}$, and that it does not follow from a weighted averaging model in which $w_{S}$ is a constant. If situational aspects affected only $S_{0}$, the lines describing the relation between tradeoff size and final conflict, $C$, would decrease with decreasing $S_{0}$ but would run strictly parallel to one another. However, it is

\footnotetext{
${ }^{1}$ These labels should not be held to imply that there are "substantive" criteria to distinguish between "categories" of differential attribute importance.
} 
A One Attribute About as Important as the Other
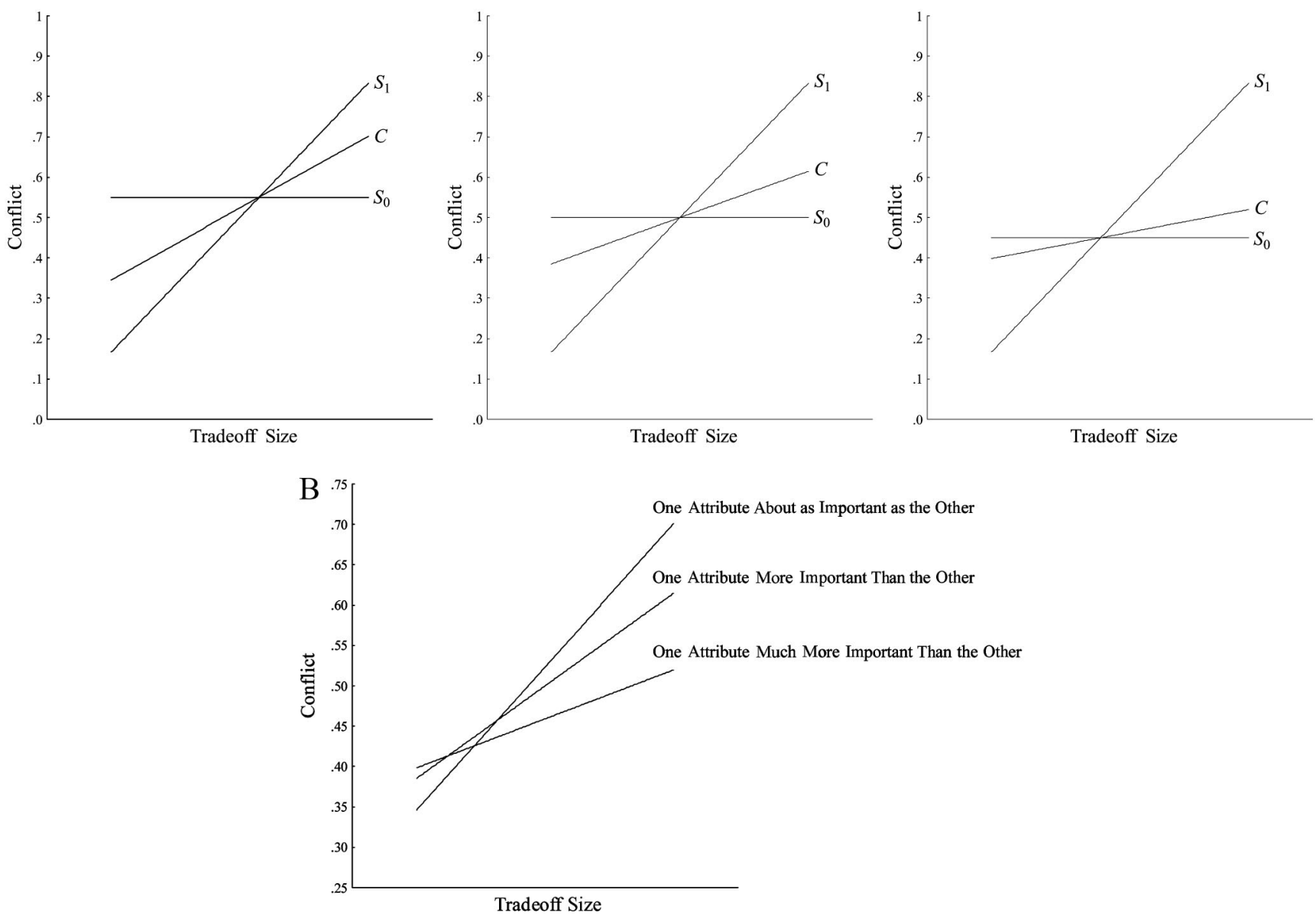

Figure 1. The single-mediation model. (A) The predicted relation between tradeoff size and conflict, as moderated by differential attribute importance. (B) Summary of the relations between tradeoff size and final conflict.

because situational aspects affect $S_{0}$ and because $w_{S}$ varies directly with $S_{0}$ that those lines gravitate toward $S_{0}$ with decreasing $S_{0}$. Ultimately, when $S_{0}$, the conflict from the preliminary impression, decreases beyond a level that it is no longer a drive for deliberation, choice will be dictated by the preliminary impression, and the relation between tradeoff size and final conflict will vanish.

As mentioned earlier, conflict is generally believed to decrease with differential attribute importance. According to the singlemediation model, this holds for the conflict from the preliminary impression, but not necessarily for final conflict. Specifically, a corollary of the above predictions, which can be seen most clearly in Figure 1B, is that final conflict may increase, rather than decrease, with differential attribute importance. This occurs when lower levels of conflict elicited by deliberation about smaller tradeoffs are losing weight relative to a decreasing but still a higher level of conflict elicited by the preliminary impression.

The single-mediation model suggests that the relation between tradeoff size and conflict is mediated only by sacrifice. Below, we develop a model of conflict generation, according to which this relation is mediated also by argumentation.

\section{Assumptions of the Double-Mediation Model}

The intuition that conflict decreases rather than increases with tradeoff size focuses on how a decision can be justified to oneself rather than what one foregoes with the decision. Festinger (1964) drew a sharp distinction between conflict before a decision, when people are supposedly impartial between the options under consideration, and cognitive dissonance after the decision, when people, motivated to justify the decision to themselves, are biased in favor of the option selected and against the option rejected, thus spreading the options apart in attractiveness. It has long been suggested, however, that self-justification also occurs before a decision, so as to make the decision possible (Janis, 1959; Shepard, 1964). More recently, the idea that people argue themselves toward a decision has reappeared under the rubric of reason-based choice (Shafir et al., 1993; Simonson, 1989). The paradigm of reason-based choice provides a rationale for a negative relation between tradeoff size and conflict. For instance, the choice between cellular phones $x_{L}=(5 \mathrm{hr}, 160 \mathrm{~g})$ and $y=(2 \mathrm{hr}, 70 \mathrm{~g})$, which makes a difference of $3 \mathrm{hr}$ in talk time and $90 \mathrm{~g}$ in weight, offers a better reason for choosing either option than the choice between 
$x_{S}=\left(2 \frac{1}{2} \mathrm{hr}, 85 g\right)$ and $y=(2 \mathrm{hr}, 70 g)$, which makes a difference of only $1 / 2 \mathrm{hr}$ in talk time and $15 \mathrm{~g}$ in weight, so that the choice between $x_{L}$ and $y$ should arouse less conflict than the choice between $x_{S}$ and $y$. In general, larger tradeoffs mean that stronger arguments can be made in favor of either decision, leading to less conflict. This proposal is incorporated into the double-mediation model, which is developed below in four steps.

Parallel updating of conflict. The double-mediation model identifies two sources of conflict in preference formation: (a) concern about sacrifice and (b) concern about argumentation. Conflict is elicited from each source by a preliminary impression of the decision problem and by subsequent deliberation about the decision problem. The conflict elicited from one source is updated in parallel with the conflict elicited from the other source.

Reverse relations with tradeoff size. The conflict elicited from the two sources by deliberation is related in opposite directions to tradeoff size. The conflict generated by concern about sacrifice is positively related to tradeoff size, because larger tradeoffs mean greater sacrifices, leading to a greater conflict, whereas the conflict generated by concern about argumentation is negatively related to tradeoff size, because larger tradeoffs mean stronger arguments, leading to less conflict.

Drives for focused deliberation. The conflict elicited from either source by the preliminary impression is, according to the double-mediation model, a drive for focused deliberation: The preliminary conflict generated by concern about sacrifice or concern about argumentation is the drive for addressing that concern during subsequent deliberation.

Aversion to conflict. Although people may generally not be oriented toward a choice that is trouble-free, this does not mean that they enjoy figuring out which choice is good enough. Conflict is actually believed to be an unpleasant state from which people wish to escape (Shepard, 1964), and support for this belief comes from research in which participants may either choose one of the options under consideration or choose none (the no-choice option). Evidence shows that the probability of choosing the no-choice option is greater in the presence than in the absence of a tradeoff (Dhar, 1997; Dhar \& Nowlis, 1999; Tversky \& Shafir, 1992) and that this probability increases with (other) manifestations of conflict, for example, decision time (Luce, 1998) and the number of thoughts for and against each option (Dhar, 1997). Thus, people do tend to escape from conflict, if there is a chance to do so. ${ }^{2}$ The double-mediation model suggests, however, that, even if there is no chance to escape, people still tend to cope with conflict along the route of least resistance. This pattern, of which escape is the most drastic manifestation, is referred to as conflict aversion.

The double-mediation model identifies two sources of conflict from which, in most cases, unequal levels of conflict will be elicited. The question arises, Will these levels of conflict contribute equally to final conflict or not? In view of conflict aversion, the answer is that they will not: The lower level of conflict, whatever its source, will contribute more to final conflict.

To illustrate, imagine that, when faced with a small tradeoff, little conflict is generated by concern about sacrifice but a lot of conflict is generated by concern about argumentation, and that, when faced with a large tradeoff, a lot of conflict is generated by concern about sacrifice but little conflict is generated by concern about argumentation. Conflict aversion means that, in the former case, the small magnitude of the sacrifices receives more attention than the weakness of the arguments (the decision tends to be treated as "trivial" rather than "very hard to justify"), whereas, in the latter case, the strength of the arguments receives more attention than the large magnitude of the sacrifices (the decision tends to be treated as "very easy to justify" rather than "highly consequential"). Alternatively, imagine that, when faced with some intermediate tradeoff, concern about sacrifice generates as much conflict as concern about argumentation. In this case, one source receives as much attention as the other (there is no route of least resistance). Thus, according to the double-mediation model, conflict aversion manifests itself as defensive inattention (Wood, 2000) to the source from which most conflict is elicited.

In sum, the double-mediation model assumes that the conflict elicited from either source by the preliminary impression is a drive for focused deliberation; that the conflict elicited from the two sources by deliberation is related in opposite directions to tradeoff size; that the conflict elicited from one source is updated in parallel with the conflict elicited from the other source; and that the lower level of conflict, whatever its source, contributes more to final conflict. A formal statement of these assumptions follows below.

\section{Formulation of the Double-Mediation Model}

The parallel updating of conflict can be captured by a pair of updating rules, one for the conflict generated by concern about sacrifice, $S$, and the other for the conflict generated by concern about argumentation, $A$ :

$$
\begin{gathered}
S=\left(1-w_{S}\right) S_{0}+w_{S} S_{1}, \\
A=\left(1-w_{A}\right) A_{0}+w_{A} A_{1},
\end{gathered}
$$

where $A_{0}, A_{1} \geq 0$ and $0 \leq w_{A}<1$. Given this formulation, the double-mediation model invokes additional assumptions about how $S_{1}$ and $A_{1}$ are related to tradeoff size, how $w_{S}$ and $w_{A}$ are related to the conflict from the preliminary impression, and how $S$ and $A$ are integrated into final conflict. First, to capture the reverse relations between tradeoff size and conflict, $S_{1}$ and $A_{1}$ are defined as functions of tradeoff size:

$$
\begin{aligned}
& S_{1}=\mathbf{f}_{S}(T), \\
& A_{1}=\mathbf{f}_{A}(T),
\end{aligned}
$$

where $\mathbf{f}_{S}$ is an increasing function and $\mathbf{f}_{A}$ is a decreasing function. In the absence of evidence to the contrary, the double-mediation model assumes no fundamental asymmetry between the mediating effects of sacrifice and argumentation, so that, whatever the size of the tradeoff, $\mathbf{f}_{S}$ increases as much as $\mathbf{f}_{A}$ decreases. For simplicity, we assume in this article that $\mathbf{f}_{S}$ and $\mathbf{f}_{A}$ are linear functions. Second, to capture the capacity of the preliminary conflict levels as drives for focused deliberation, $w_{S}$ and $w_{A}$ are defined as a function of $S_{0}$ and $A_{0}$ respectively:

$$
\begin{aligned}
& w_{S}=\mathbf{g}\left(S_{0}\right), \\
& w_{A}=\mathbf{g}\left(A_{0}\right),
\end{aligned}
$$

where $\mathbf{g}$ is the same increasing function in both equations. Third, given conflict aversion, the relative contribution of $S$ and $A$ to final

\footnotetext{
${ }^{2}$ The whole trajectory, then, is one of getting ready (orientation reaction), getting stuck (vacillation), and getting out (escape).
} 
conflict is a function of their relative levels, in that the lower level contributes more. This can be captured by a differentially weighted averaging model:

$$
C=(1-w) S+w A,
$$

where $0 \leq w \leq 1,1-w$ is the relative weight of $S, w$ is the relative weight of $A$, and $w$ is defined as a function of $S$ and $A$ :

$$
w=\mathbf{h}(S, A),
$$

where $\mathbf{h}$ is a function with three properties: It is increasing in $S$, it is decreasing in $A$, and $\mathbf{h}\left(C_{S}, C_{A}\right)=1 / 2$ if and only if $S=A$. If the latter holds, then $w=1-w=1 / 2$, so that $S$ and $A$ contribute equally to $C$. However, if $S>A$, then $w>1 / 2$, so that $A$ contributes more to $C$, but if $S<A$, then $1-w>1 / 2$, so that $S$ contributes more to $C$. Thus, Equation 5 changes Equation 4 into a differentially weighted averaging model, in that the relative weight of the conflict from a source is inversely related to its level: The conflict from a particular source curtails its own contribution to final conflict by raising defensive inattention to that source.

In the appendix, we provide a fully parametric formulation of the double-mediation model by specifying the functions $\mathbf{f}_{S}, \mathbf{f}_{A}, \mathbf{g}$, and $\mathbf{h}$, and we estimate its parameters from our experimental results. (We also perform parametric tests of our hypotheses.) That formulation and those parameters are the blueprint for all the figures illustrating the implications of the double-mediation model for the relation between tradeoff size and conflict. However, nothing in the appendix is indispensable for understanding those implications.

\section{Implications of the Double-Mediation Model}

The double-mediation model predicts that conflict will generally have an inverse $U$-shaped relation with tradeoff size but that this relation will be moderated by other aspects of the decision situation. In Experiment 1, we concentrate on differential attribute importance, as mentioned earlier.

The left panel of Figure 2A shows a situation in which one attribute is about as important as the other. In the range of small to intermediate tradeoffs, concern about sacrifice generates less conflict than concern about argumentation, whereas, in the range of intermediate to large tradeoffs, concern about argumentation generates less conflict than concern about sacrifice. In view of conflict aversion, lower levels of

A One Attribute About as Important as the Other

One Attribute More Important Than the Other

One Attribute Much More Important Than the Other

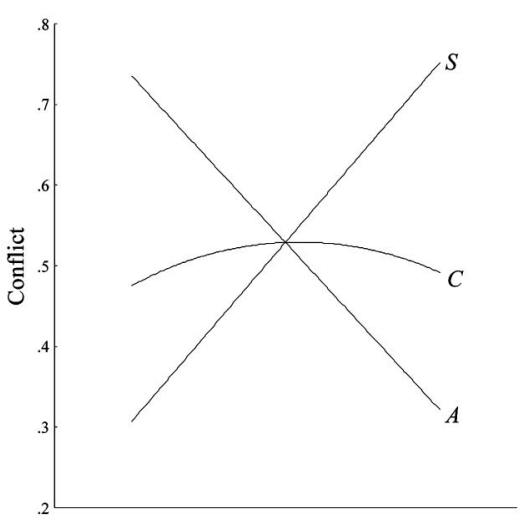

Tradeoff Size

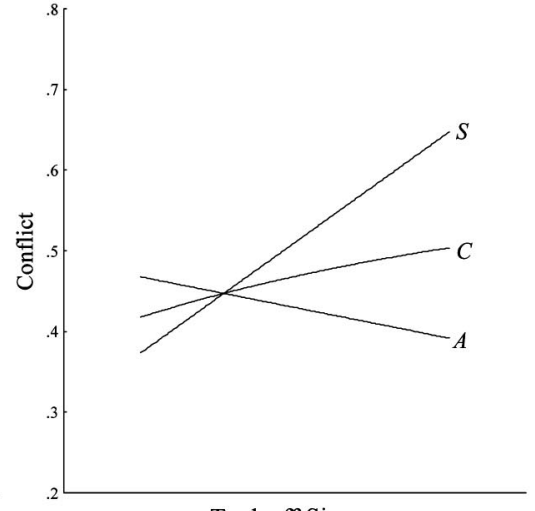

Tradeoff Size

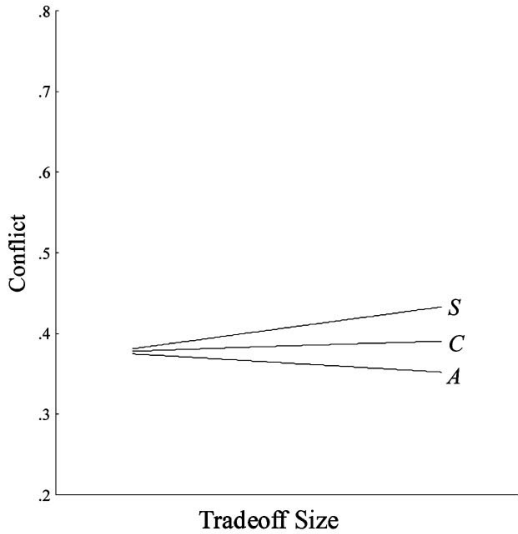

Tradeoff Size

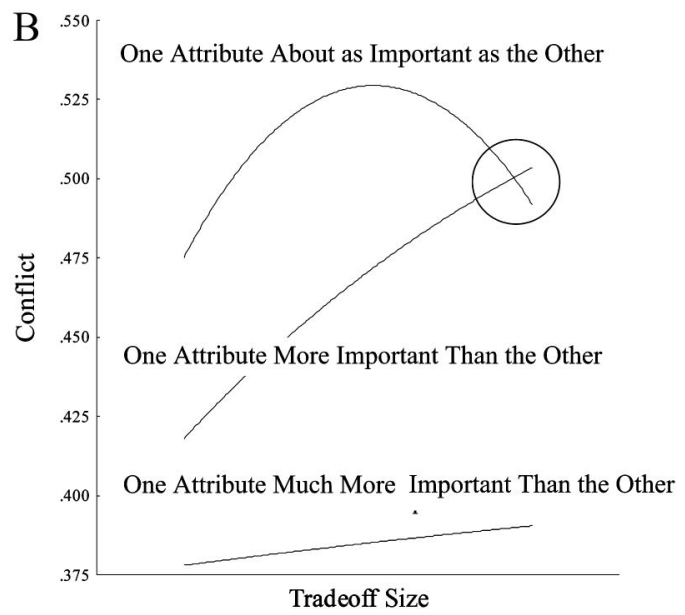

Figure 2. The double-mediation model. (A) The predicted relation between tradeoff size and conflict, as moderated by differential attribute importance. (B) Summary of the relations between tradeoff size and final conflict. 
conflict, whatever their source, contribute more to final conflict. Therefore, in the range of smaller tradeoffs, the conflict generated by concern about sacrifice, which has a positive relation with tradeoff size, contributes more to final conflict whereas, in the range of larger tradeoffs, the conflict generated by concern about argumentation, which has a negative relation with tradeoff size, contributes more to final conflict. Overall, then, final conflict has an inverse $U$-shaped relation with tradeoff size.

We emphasize that the inverse $U$-shaped relation follows only from the differentially weighted averaging model, in which the relative contribution of the conflict from the two sources to final conflict is a function of their relative levels, and that it does not follow from a weighted averaging model in which their relative contribution is constant. To illustrate, in the left panel of Figure 2A, the reverse relations between tradeoff size and the conflict from the two sources form a (nearly) symmetrical $X$ pattern. In the absence of conflict aversion, and in the absence of any good reason why the conflict from one source would contribute more to final conflict than the conflict from the other source, the relation between tradeoff size and final conflict would be a (nearly) flat line.

What are the implications of a greater difference in importance between the attributes? First, the double-mediation model predicts that differential attribute importance will lead to a decrease in the preliminary conflict generated by concern about sacrifice, because incurring a sacrifice along the less important attribute seems less painful than incurring a sacrifice along the more important attribute. Second, the double-mediation model predicts that differential attribute importance will lead to an even greater decrease in the preliminary conflict generated by concern about argumentation, because it offers the decision maker a strong argument for a particular decision (Shafir et al., 1993; Slovic, 1975; Tversky, Sattath, \& Slovic, 1988), but it offers no relief from the sacrifice to be incurred in making that decision (less painful as that sacrifice may seem than the sacrifice to be incurred in making the opposite decision). Third, the double-mediation model predicts that a fall in preliminary conflict generated by concern about sacrifice or concern about argumentation will attenuate the relation between tradeoff size and the conflict generated by that concern, because it decreases the associated drive for focused deliberation.

The center panel of Figure 2A shows a situation in which one attribute is more important than the other. The relation between tradeoff size and the conflict generated by each concern has been attenuated, but the negative relation between tradeoff size and the conflict generated by concern about argumentation has been attenuated to a greater degree than the positive relation between tradeoff size and the conflict generated by concern about sacrifice. As a result, the relation between tradeoff size and final conflict has changed in an upward direction. ${ }^{3}$ More specifically, the inverse $\mathrm{U}$-shaped relation has changed into a positive relation.

Finally, what are the implications of a still greater difference in importance between the attributes? According to the doublemediation model, the conflict from the preliminary impression will decrease even further and, with it, the drives for focused deliberation, so that the relation between tradeoff size and conflict will be attenuated even further. The right panel of Figure 2A shows a situation in which one attribute is much more important than the other. As can be seen, the relation between tradeoff size and final conflict has changed in a downward direction. More specifically, the positive relation has changed into a nearly zero relation.
In sum, we arrive at the following hypotheses about how conflict is related to the size of the tradeoff between the attributes under consideration and how this relation is moderated by the difference in importance between the attributes:

H1.1. When one attribute is about as important as the other (equal attribute importance), there will be an inverse $\mathrm{U}$-shaped relation between tradeoff size and conflict;

H1.2. When one attribute is more important than the other (unequal attribute importance), the relation between tradeoff size and conflict will change in an upward direction, more specifically, the inverse $U$-shaped relation will change into a (more) positive relation;

H1.3. When one attribute is much more important than the other (very unequal attribute importance), the relation between tradeoff size and conflict will change in a downward direction, more specifically, the positive relation will change into a (nearly) zero relation.

Conflict is generally believed to decrease with differential attribute importance (for support, see Chatterjee \& Heath, 1996; Scholten, 2002; Simonson, 1989). According to the doublemediation model, this holds for the conflict from the preliminary impression but not necessarily for final conflict. As can be seen in Figure $2 \mathrm{~B}$, the final conflict aroused by large tradeoffs is, in this example, greater in the situation of unequal attribute importance than in the situation of equal attribute importance (the cross-over point is marked by a circle), although final conflict is generally lower in the former situation than in the latter.

\section{Measuring Conflict}

There is no generally accepted procedure for measuring conflict (Tversky \& Shafir, 1992). Actually, the literature suggests a large variety of mental and behavioral manifestations of conflict. In our experiments, we derive a composite measure of conflict, denoted $\hat{C}$ from the following five component measures, all of which are positively related to conflict ${ }^{4}$ :

1. The time to reach a decision (Berlyne, 1957a; Diederich, 2003; Festinger, 1943a, 1943b; Fischer, Jia, \& Luce, 2000a; Fischer, Luce, \& Jia, 2000b; Kiesler, 1966; Luce, 1998; Luce, Bettman, \& Payne, 1997; Tyebjee, 1979), denoted $t$;

2. The degree to which the options are equally preferred (Tyebjee, 1979), denoted $E(v)$;

\footnotetext{
${ }^{3}$ Statements that a relation changes in an upward or a downward direction concern changes in the linear trend of the relation and cover a great diversity of situations. For instance, when an inverse U-shaped relation changes into a positive relation, the linear trend changes from (nearly) zero to greater than zero. Alternatively, when a negative relation changes into an inverse $\mathrm{U}$-shaped relation, the linear trend changes from smaller than zero to (nearly) zero. The statement that the relation changes in an upward direction applies to both situations.

${ }^{4}$ Other measures of conflict used in the literature, but not included in our composite measure of conflict, are the number of thoughts for and against each option under consideration (Dhar, 1997; Simonson, 1989), choice of the no-choice option (Dhar, 1996, 1997; Dhar \& Nowlis, 1999; Dhar \& Simonson, 2003; Luce, 1998; Tversky \& Shafir, 1992), anticipated regret about the decision reached (Chatterjee \& Heath, 1996), and inconsistency between decisions reached on separate occasions (Fischer et al., 2000a, 2000b; Luce, Jia, \& Fischer, 2003).
} 
3. The degree to which the attributes are equally important for the decision (being) reached (Simonson, 1989), denoted $E(w)^{5}$;

4. The difficulty of reaching a decision (Chatterjee \& Heath, 1996; Scholten, 2002; Simonson, 1989), denoted $d$; and

5. The lack of confidence in the (correctness of the) decision reached (Dhar, 1996; Festinger, 1943a, 1943b; Russo, Meloy, \& Medvec, 1998; Zakay, 1985; Zakay \& Tsal, 1993), denoted $u$.

There are at least three differences between decision time and the other conflict measures as we obtain them. Decision time is a behavioral manifestation of conflict, recorded unobtrusively by the experimenter as a decision is being reached ("online"). The other conflict measures are mental manifestations of conflict, rated by the participants (along standard rating scales) after a decision has been reached ("off-line"). ${ }^{6}$ The methodological differences between decision time and the rating measures of conflict are useful for assessing the construct validity of composite conflict measure $\hat{C}$.

\section{Experiment 1}

\section{Method}

Design and stimuli. Each participant completed 12 decision tasks, four tasks in which, according to pretest results, one attribute was about as important as the other (equal attribute importance), four tasks in which one attribute was more important than the other (unequal attribute importance), and four tasks in which one attribute was much more important than the other (very unequal attribute importance). The more important attribute is denoted attribute 1 and the less important attribute is denoted attribute $2 .^{7}$

In addition to differential attribute importance, tradeoff size was manipulated across the 12 decision tasks. Each participant completed four tasks in which, also according to pretest results, the difference between the options along each attribute was perceived to be small and insignificant (small tradeoff), four tasks in which the difference between the options along each attribute was perceived to be large and significant (large tradeoff), and four tasks in which the perceived difference between the options along each attribute fell between these two extremes (intermediate tradeoff). So that any particular decision task would not always be associated with any particular tradeoff size, the association of tradeoff sizes with decision tasks was counterbalanced across three groups of participants. For one group, a particular task would be associated with a small tradeoff; for another group, this same task would be associated with an intermediate tradeoff; and for the remaining group, it would be associated with a large tradeoff.

Independently of tradeoff size, the option with respect to which tradeoffs were small, intermediate, or large was manipulated between participants, in that tradeoff size varied with respect to the option that was superior along attribute 1 , denoted option $x$, for one half of the participants and with respect to the option that was superior along attribute 2, denoted option $y$, for the other half (see Figure 3). ${ }^{8}$ This control variable had no reliable effects on conflict and will not be discussed further.

As shown in Figure 3, the options composing the dyadic choice sets were located along a straight line in attribute space (up to slight deviations from that line where attribute amounts were rounded), so that the rate of exchange between the attributes was (practically) constant across tradeoffs of different size. Table 1 provides a sample of the stimulus material used in Experiment 1.

Participants and procedure. A total of 100 psychology students participated in the experiment and were paid €7.50. Experimental sessions were run by computer with at most 10 participants at a time. The participants were informed that they would complete 12 decision tasks, each accompanied by 4 rating tasks. They were given a description of these tasks as well as instructions and rehearsal trials for keyboard handling. Before the session began, they were asked to provide thoughtful and truthful responses, relying on their personal tastes and judgments.
The order of the 12 decision tasks was randomized across participants. Each task was displayed in an attribute (row) by option (column) format (see Figure 4). The left-right position of options $x$ and $y$ as well as the top-down position of attributes 1 and 2 were randomized across participants and decision tasks. The options were labeled $P$ and $Q$ from left to right, except when the task was selecting a laser or inkjet printer (see also Table 1) or selecting a car on natural gas or gasoline, in which cases the options were labeled as such. Before an option was selected, a leftward arrow was located below the left option and a rightward arrow was located below the right option, as shown in the top panel of Figure 4. The participant selected an option by pressing the leftward or rightward arrow on the keyboard. After that, the arrow below the nonselected option disappeared, the arrow below the selected option remained (now blinking), and on the bottom of the screen appeared a reminder that the participant should press "Backspace" to correct the decision or "Enter" to confirm it, as shown in the bottom panel of Figure 4. Depending on the participant's response, the program either returned to the original display of the decision task or proceeded to the first rating task. Decision time was counted in milliseconds from the moment that the decision task appeared on the screen until the moment that the participant confirmed the decision.

Each decision task was accompanied by four rating tasks. The participants rated the preference between the options, the importance of the attributes for the decision reached, the difficulty of reaching a decision, and the (lack of) confidence in the correctness of the decision reached. The order of the rating tasks was randomized across participants and decision tasks.

Figure 5 shows how a rating task was displayed. The attribute-by-option information as well as the decision reached on the basis of that information were presented on the top of the screen. A 9-point rating scale was presented on the bottom. Before a rating was given, numbers were located above the 9 positions of the scale, as shown in the top panel of Figure 5. The participant selected a position by pressing one of the corresponding digits on the keyboard. After that, the numbers above the nonselected positions disappeared, the number above the selected position remained (now blinking), and on the bottom of the screen appeared a reminder that the participant should press "Backspace" to correct the rating or "Enter" to confirm it, as shown in the bottom panel of Figure 5. Depending on the participant's response, the program either returned to the original display of the rating task or proceeded to the following one. We also recorded rating times, counted in milliseconds from the moment that a rating task appeared on the screen until the moment that the participant confirmed a rating.

\footnotetext{
${ }^{5}$ In the measurement context, $w$ refers to the relative weight of the attributes, not, as in Equation 4, to the relative weight of the conflict generated by concern about sacrifice and the conflict generated by concern about argumentation in determining final conflict. The meaning of $w$ should be clear from the context.

${ }^{6}$ The degree to which the attributes are equally important for the decision reached, conflict measure $E(w)$, is conceptually distinct from the degree to which one attribute is more important than the other before any decision is being reached, the moderator variable of Experiment 1, in that the former derives from a local appraisal of attribute importance, which may be affected by the differences between the options along the attributes, whereas the latter involves a global appraisal of attribute importance, which is unaffected by the set of options under consideration (a distinction introduced by Goldstein, 1990)

${ }^{7}$ Naturally, the designation of "equal" attribute importance is an approximation: No pair of attributes is exactly equal in importance. This is appropriately expressed by the alternative designation that one attribute is "about" as important as the other.

${ }^{8}$ Chatterjee and Heath (1996) manipulated tradeoff size by varying the deviation of the options from their midpoint along the attributes. We do not use this manipulation, because it confounds the size of the tradeoff between the attributes with the extremeness of the options along those attributes.
} 


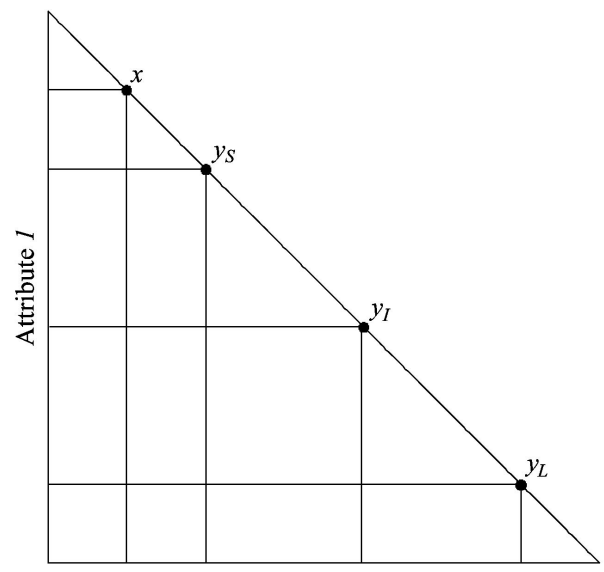

Attribute 2

The option with respect to which tradeoffs are small,

intermediate, or large is option $x$, which is superior

along attribute 1 .

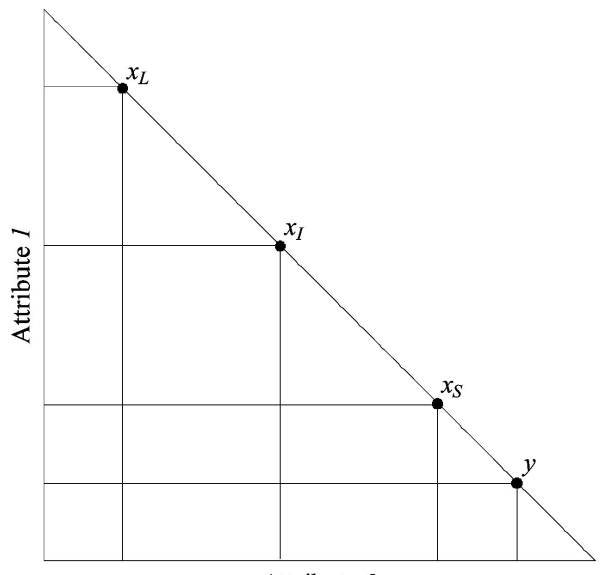

Attribute 2

The option with respect to which tradeoffs are small,

intermediate, or large is option $y$, which is superior

along attribute 2 .

Figure 3. The manipulation of tradeoff size. In the left panel, $y_{s}, y_{l}$, and $y_{L}$, are options that imply a small, intermediate, and large tradeoff, respectively, with option $x$. Similarly, in the right panel, $x_{s}, x_{I}$, and $x_{L}$ are options that imply a small, intermediate, and large tradeoff, respectively, with option $y$. The large tradeoff in the left panel is the same as the one in the right panel. The attributes are scaled from worse to better.

The preference rating scale was presented as in Figure 5. The other rating scales were presented in a similar fashion. Along the importance rating scale, positions $1,3,5,7$, and 9 were labeled much more important, more important, equally important, more important, and much more important, respectively. The left-right position of the attributes above this scale corresponded to their top-bottom position elsewhere. Along the difficulty rating scale, positions $1,3,5,7$, and 9 were labeled very easy, easy, neither easy nor difficult, difficult, and very difficult, respectively. Along the confidence rating scale, positions 1 and 9 were labeled no confidence and full confidence, respectively.

Measures. All response times (decision and rating times) were converted from milliseconds to seconds. Characteristically, the response times had positively skewed distributions, so that their means would be unduly affected by relatively few, very slow responses. A logarithmic transformation of the response times largely removed the positive skew from all distributions. Thus, conflict measure $t$, the time to reach a decision, became $\log _{2}(t)$, the logarithm of decision time. Similarly, rating times were transformed to $\log _{2}\left(t_{v}\right), \log _{2}\left(t_{w}\right), \log _{2}\left(t_{d}\right)$ and $\log _{2}\left(t_{u}\right)$ for preference, importance, difficulty, and confidence, respectively.

The preference ratings, $v$, and importance ratings, $w$, were coded from $1 / 10$ to $9 / 10$. Conflict measures $E(v)$, the degree to which the options were equally preferred, and $E(w)$, the degree to which the attributes were equally important for the decision reached, were obtained by applying an entropy measure to $v$ and $w: E(v)=-\left[v \log _{2}(v)+(1-v) \log _{2}(1-v)\right]$ and $E(w)=-\left[w \log _{2}(w)+(1-w) \log _{2}(1-w)\right]$, where $0<v, w<1$. To illustrate, when the options are equally preferred, that is, $v=1 / 2$, conflict is at its highest level, i.e., $E(v)=1$. As the preference for one option over the other increases, that is, $v \rightarrow 0$ or $v \rightarrow 1$, conflict decreases toward its lowest level, that is, $E(v) \rightarrow 0$, and at an accelerating rate. An entropy measure of conflict was explored by Berlyne (1957b) and applied to preference ratings by Tyebjee (1979).

The difficulty ratings were coded from 1 (very easy) to 9 (very difficult), thus yielding conflict measure $d$, the difficulty of reaching a decision. Similarly, the confidence ratings were coded from 1 (full confidence) to 9 (no confidence), thus yielding conflict measure $u$, the lack of confidence in the correctness of the decision reached.

\section{Results}

Differential attribute importance: Manipulation check. A check on the manipulation of differential attribute importance was conducted by comparing the probability of choosing option $x$ (the option that was superior along the more important attribute) between the three conditions of differential attribute importance. This probability was significantly lower when one attribute was about as important as the other (.59) than when one attribute was more important than the other (.77), $z=-5.36, p=.00$ and, in turn, significantly lower when one attribute was more important than the other (.77) than when one attribute was much more important than the other (.82), $z=-1.92, p=.03 .{ }^{9}$ Thus, differential attribute importance was manipulated as intended.

Test of hypotheses: Dependent variable. Table 2 provides the means, standard deviations, and correlations for all measures taken in Experiment 1. We conducted a principal component analysis on the five conflict measures across the 100 participants and the 12 decision tasks. The first component had an eigenvalue of $\lambda=2.85$, thus accounting for $57.07 \%$ of the total variance. No other eigenvalues exceeded the unit threshold. The reliability of the unweighted composite of standardized conflict measures was $\alpha=$ .81. To improve reliability, we constructed a weighted composite measure of conflict, $\hat{C}$, by deriving the (standardized) scores on the first component (see Lindeman, Merenda, \& Gold, 1980, pp. 281-283) and then linearly transforming these scores to a scale from 0 to 1 .

\footnotetext{
${ }^{9}$ Newman-Keuls tests revealed that there were no significant differences in the probability of choosing option $x$ among the decision tasks representing the same level of differential attribute importance.
} 
Table 1

Sample of the Stimulus Material Used in Experiment 1

\begin{tabular}{|c|c|c|c|c|c|c|}
\hline \multirow[b]{2}{*}{ Domain and attributes ${ }^{\mathrm{b}}$} & \multicolumn{6}{|c|}{ Dyadic choice sets $\{x, y\}^{\mathrm{a}}$} \\
\hline & $x$ & $y_{s}$ & $y_{\mathrm{I}}$ & $x_{\mathrm{I}}$ & $x_{s}$ & $y$ \\
\hline \multicolumn{7}{|l|}{ Cellular phones } \\
\hline \multirow{5}{*}{1 Talk time (h:min) } & $5: 00$ & $4: 20$ & & & & \\
\hline & $5: 00$ & & 3:00 & & & \\
\hline & & & & & & $2: 00$ \\
\hline & & & & 4:00 & & 2:00 \\
\hline & & & & & $2: 30$ & $2: 00$ \\
\hline \multirow[t]{5}{*}{2 Weight $(g)$} & 160 & 140 & & & & \\
\hline & 160 & & 100 & & & \\
\hline & 160 & & & & & 70 \\
\hline & & & & 130 & & 70 \\
\hline \multirow{2}{*}{\multicolumn{7}{|c|}{ Laser $(x)$ or inkjet $(y)$ printer }} \\
\hline & & & & & & \\
\hline \multirow[t]{5}{*}{1 Cost per print (cents) } & 1 & 2 & & & & \\
\hline & 1 & & 5 & & & \\
\hline & 1 & & & & & 10 \\
\hline & & & & $71 / 2$ & & 10 \\
\hline & & & & & 9 & 10 \\
\hline \multirow[t]{5}{*}{2 Price $(€)$} & 700 & 645 & & & & \\
\hline & 700 & & 480 & & & \\
\hline & 700 & & & & & 200 \\
\hline & & & & 340 & & 200 \\
\hline & & & & & 255 & 200 \\
\hline \multicolumn{7}{|l|}{ Cars } \\
\hline \multirow[t]{4}{*}{1 Mileage (L/100 km) } & 5 & 6 & & & & \\
\hline & 5 & & 8 & & & \\
\hline & 5 & & & & & 14 \\
\hline & & & & 10 & & 14 \\
\hline \multirow{5}{*}{$\begin{array}{l}2 \text { Acceleration (s to reach } \\
100 \mathrm{~km} / \mathrm{h} \text { ) }\end{array}$} & 17 & 16 & & & & \\
\hline & 17 & & 14 & & & \\
\hline & 17 & & & & & 9 \\
\hline & & & & 13 & & 9 \\
\hline & & & & & 10 & 9 \\
\hline
\end{tabular}

Note. Complete listings of the stimulus material can be obtained from Scholten and Sherman (2004).

${ }^{a}$ Small tradeoffs are implied by $\left\{x, y_{s}\right\}$ and $\left\{x_{s}, y\right\}$. Intermediate tradeoffs are implied by $\left\{x, y_{\mathrm{I}}\right\}$ and $\left\{x_{\mathrm{I}}, y\right\}$. A large tradeoff is implied by $\{x, y\}$.

${ }^{\mathrm{b}}$ Examples from the experimental conditions in which Attribute 1 was about as important as Attribute 2 (cellular phones), Attribute 1 was more important than Attribute 2 (laser or inkjet printer), and Attribute 1 was much more important than Attribute 2 (cars), respectively.

Test of hypotheses: Independent variables. The hypotheses were tested with linear regression analyses, run across participants and decision tasks. Pooling across $k$ decision tasks is common practice in related research where the dependent variable is dyadic choice (rather than the conflict aroused by the choice). Usually, the pooled regression analyses conducted in that research either include $k-1$ interactions between an independent variable and the $k$ decision tasks so as to check whether the effect of the variable is robust across the tasks (Dhar, 1997; Dhar \& Nowlis, 2004; Dhar \& Sherman, 1996; Dhar \& Simonson, 2003) or include $2 k-1$ alternative-specific dummies so as to capture the overall probability with which each option is chosen (Dhar \& Nowlis, 1999; Nowlis, 1995; Nowlis \& Simonson, 1996). We adapted both approaches to our regression analyses.

We conducted 4 regression analyses, one across the 12 decision tasks, representing 3 levels of differential attribute importance and 4 replications of each level, and 3 analyses across the 4 replications within each level of differential attribute importance. The regression analysis across the 12 decision tasks addressed the 3 hypotheses combined (H1.1-H1.3), evaluating the changing shape of the relation between tradeoff size and conflict with changes in differential attribute importance. The analysis included 2 orthogonal polynomial contrasts (linear and quadratic) between the 3 levels of differential attribute importance, 9 orthogonal difference contrasts (or "inverse Helmert" contrasts) between the 4 replications of the respective levels of differential attribute importance (capturing the overall differences in conflict between replications), 2 orthogonal polynomial contrasts (linear and quadratic) between the 3 levels of tradeoff size, 1 contrast between the 2 levels of the control variable (the option with respect to which tradeoff size was varied), and 12 orthogonal contrasts capturing the interactions between the independent variables (differential attribute importance, tradeoff size, and the control variable).

The 3 analyses across the 4 replications within each level of differential attribute importance addressed the 3 hypotheses individually, evaluating the specific shapes of the relation between tradeoff size and conflict as well as their robustness across decision tasks. Each analysis included 3 orthogonal difference contrasts between the 4 replications of the particular level of differential attribute importance (capturing the overall differences in conflict between replications), 2 orthogonal polynomial contrasts (linear and quadratic) between the 3 levels of tradeoff size, 1 contrast between the 2 levels of the control variable (the option with respect to which tradeoff size was varied), and 2 orthogonal contrasts capturing the interaction between the independent variables (tradeoff size and the control variable). To check whether the linear or quadratic trend in the relation between tradeoff size and conflict was robust across decision tasks, we evaluated whether inclusion of 3 orthogonal contrasts capturing the interaction between trend and decision task yielded a reliable and meaningful increase in goodness-of-fit $\left(R^{2}\right)$ of the regression model.

Tests of hypotheses: Results. Figure 6 shows how the relation between tradeoff size and conflict is moderated by differential attribute importance. Two regression results confirmed the changing shape of the relation between tradeoff size and conflict with changes in differential attribute importance. First, the linear contrast between the levels of tradeoff size interacted significantly with the quadratic contrast between the levels of differential attribute importance, $t(1173)=2.58, p=.01$ : Consistent with H1.1-H1.3, the relation between tradeoff size and conflict was more positive when one attribute was more important than the other than when one attribute was about as important as the other or when one attribute was much more important than the other. Second, the quadratic contrast between the levels of tradeoff size interacted significantly with the linear contrast between the levels of differential attribute importance, $t(1173)=-3.00, p=.00$ : Consistent with H1.1, there was an inverse U-shaped relation between tradeoff size and conflict when one attribute was about as important as the other but, inconsistent with H1.3, there was a $U$-shaped relation between tradeoff size and conflict rather than a (nearly) zero relation, when one attribute was much more important than the other.

Two other results emerged from the same regression analysis. First, the linear contrast between the levels of differential attribute importance was significant, $t(1173)=-4.15, p=.00$ : Consistent with existing evidence, conflict generally decreased with differential attribute importance. Second, the linear contrast between the 


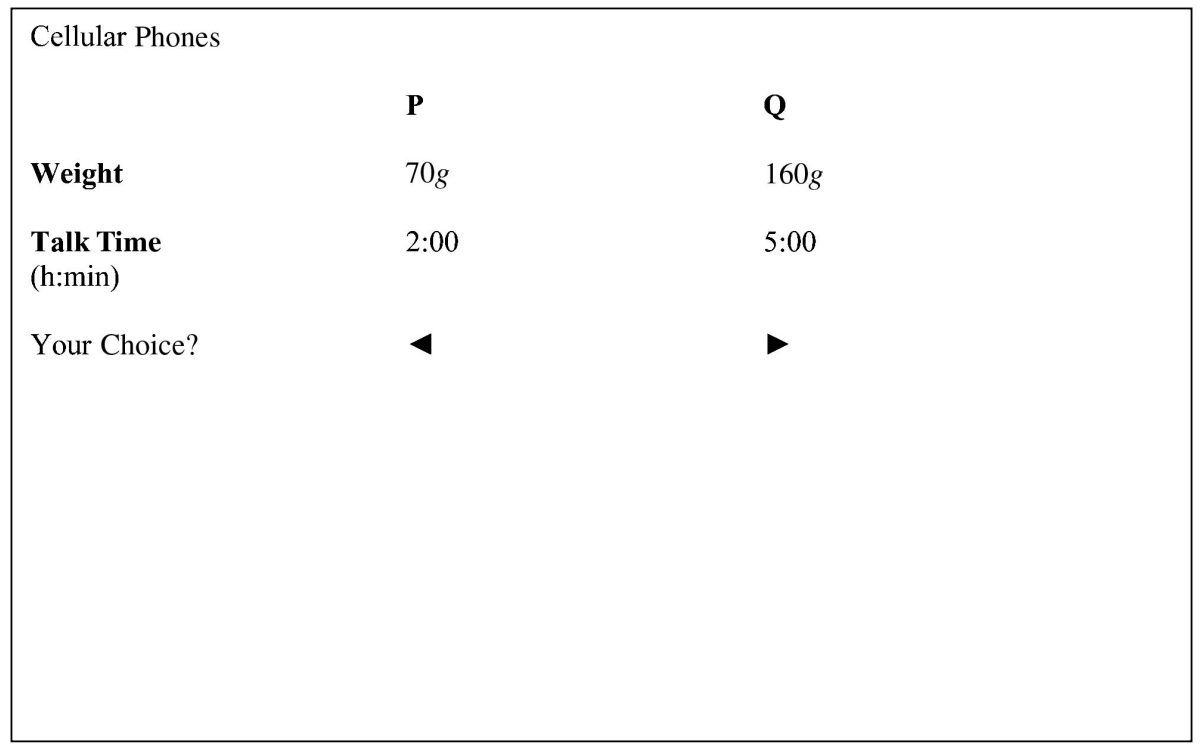

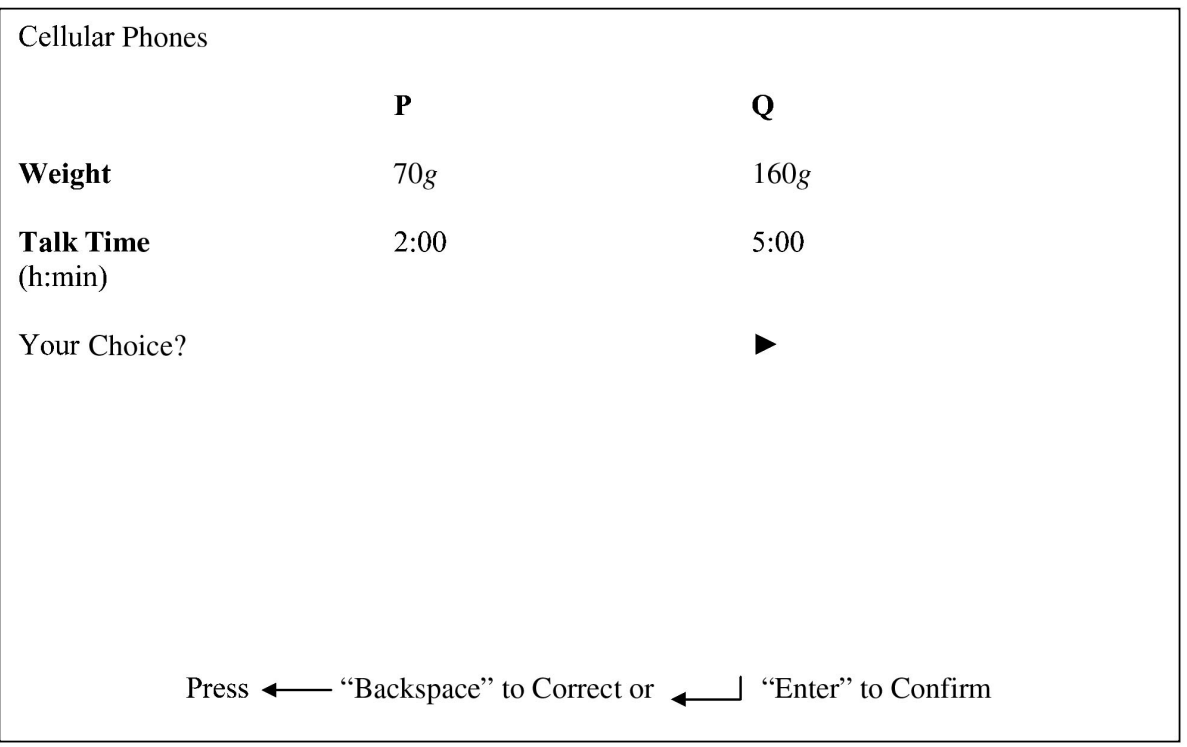

Figure 4. Display of each decision task on the computer screen, before (top panel) and after (bottom panel) the selection of an option.

levels of tradeoff size was significant, $t(1173)=2.60, p=.01$ : Conflict generally increased with tradeoff size. Most theories of decision making view this result as fundamental; the doublemediation model views it as incidental to the decision situations that are being considered.

Three regression results confirmed the specific shapes of the relation between tradeoff size and conflict as well as their robustness across decision tasks. First, when one attribute was about as important as the other, the quadratic contrast between the levels of tradeoff size was significant, $t(391)=2.30, p=.02$ : Consistent with H1.1, there was an inverse $\mathrm{U}$-shaped relation between tradeoff size and conflict. Including the interaction between quadratic trend and decision task yielded an increase in goodness-offit that was very small, .087 - .084 $=.002$, and insignificant, $F(8$, 11) $=0.78, p=.63$. Thus, the inverse $\mathrm{U}$-shaped relation was robust across decision tasks.
Second, when one attribute was more important than the other, the linear contrast between the levels of tradeoff size was significant, $t(391)=3.76, p=.00$ : Consistent with H1.2, there was a positive relation between tradeoff size and conflict. Including the interaction between linear trend and decision task yielded an increase in goodness-of-fit that was very small, .177- .176 $=.002$, and insignificant, $F(8,11)=0.97, p=.50$. Thus, the linear relation was robust across decision tasks.

Third, when one attribute was much more important than the other, the quadratic contrast between the levels of tradeoff size approached significance, $t(391)=-1.89, p=.06$ : Inconsistent with $H 1.3$, there was a $\mathrm{U}$-shaped relation between tradeoff size and conflict. Including the interaction between quadratic trend and decision task yielded an increase in goodness-of-fit that was very small, .065 -.063 $=.002$, and insignificant, $F(8$, 11) $=0.71, p=.68$. Thus, the $U$-shaped relation was robust 


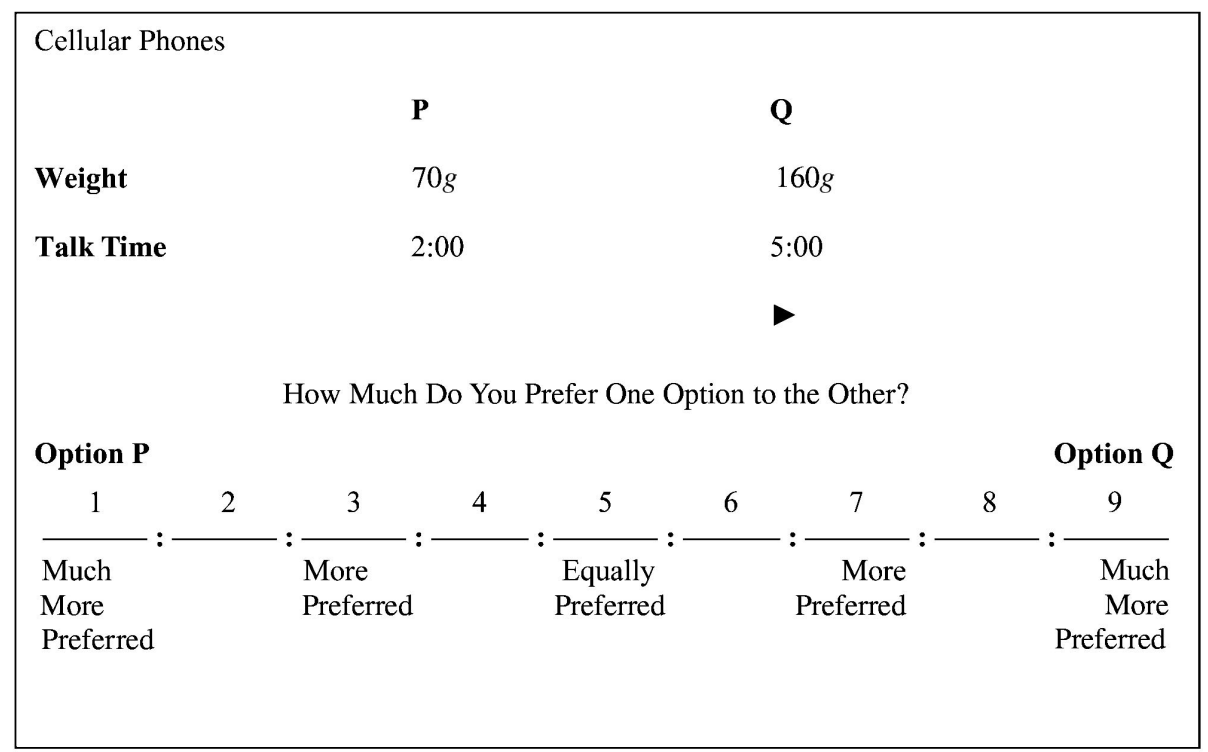

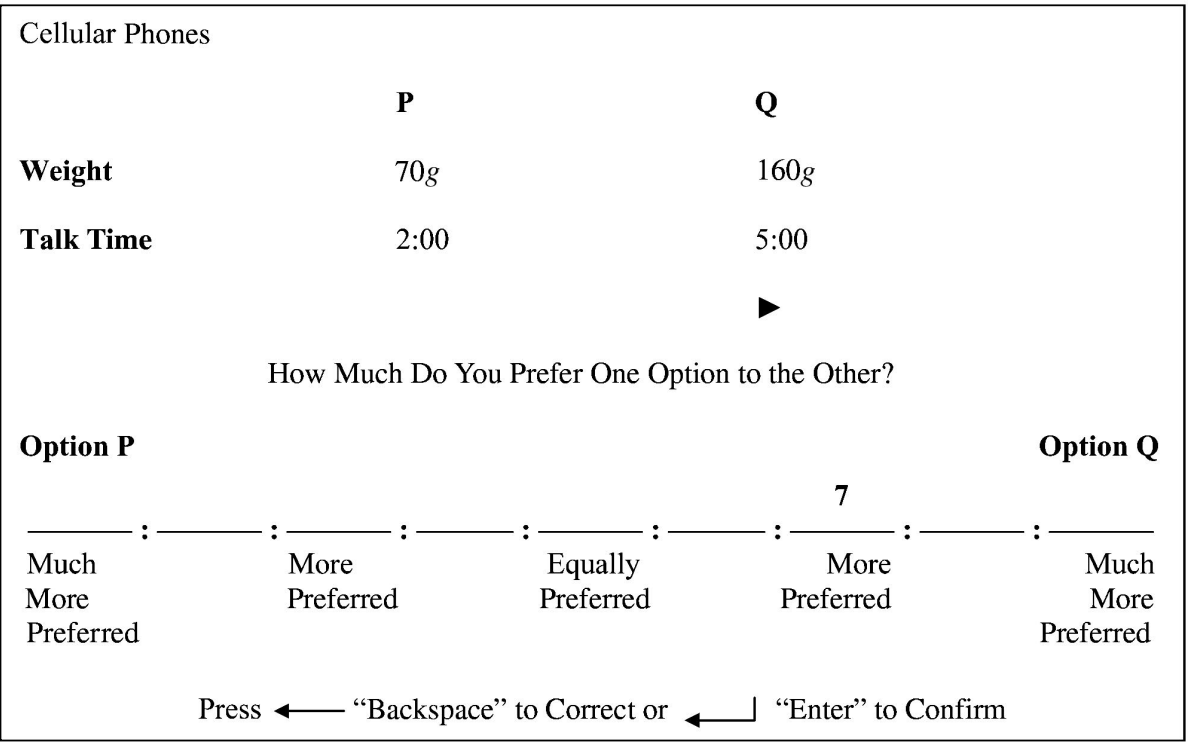

Figure 5. Display of a rating task on the computer screen, before (top panel) and after (bottom panel) the selection of a position along the rating scale.

across decision tasks. We return to this result in the "Discussion."

Conflict: Convergent and discriminant validity. As mentioned earlier, the methodological differences between decision time and the rating measures of conflict are useful for assessing the construct validity of composite conflict measure $\hat{C}$ (see also Table 2 ).

Convergent validity may be evaluated in terms of the monotrait-heteromethod correlation (see Campbell \& Fiske, 1959) between decision time and a composite of the rating measures (in principal component scores). This correlation was moderately high, $r=.42$, and significant, $t(1198)=16.14, p=$ .00 . To check whether there was any indication of an inverse $U$-shaped relation between conflict and decision time, a possibility raised by Berlyne $(1962,1966)$, we conducted a linear and a polynomial (quadratic) regression analysis with decision time as dependent variable and the composite of the rating measures as independent variable. There was no inverse $U$-shaped relation but rather a positive, slightly accelerating one. The quadratic model yielded an increase in goodness-of-fit that was significant, $F(1,1197)=18.56, p=.00$, but small, $.19-.18=$ .01. Thus, nonlinearity posed no meaningful threat to the validity of decision time as a measure of conflict.

Discriminant validity may be evaluated in terms of the difference between, on the one hand, the monotrait-heteromethod correlation between decision time and the composite of the rating measures and, on the other hand, the heterotrait-monomethod correlation between decision time and a composite of the rating times (in principal component scores). This difference was positive, $.42-.35=.07$, and significant, $t(1197)=2.37, p=.02$ (by Hotelling's $t$-statistic; see Lindeman et al., 1980, pp. 52-53). 
Table 2

Experiment 1: Means, Standard Deviations, and Correlations for Conflict Measures and Rating Times

\begin{tabular}{|c|c|c|c|c|c|c|c|c|c|c|}
\hline Variable & $M$ & $S D$ & $\log _{2}(t)$ & $E(v)$ & $E(w)$ & $d$ & $u$ & $\log _{2}\left(t_{v}\right)$ & $\log _{2}\left(t_{w}\right)$ & $\log _{2}\left(t_{d}\right)$ \\
\hline $\log _{2}(t)$ & 4.15 & 0.77 & & & & & & & & \\
\hline$E(v)$ & .81 & .17 & .30 & & & & & & & \\
\hline$E(w)$ & .77 & .19 & .26 & .59 & & & & & & \\
\hline$d$ & 4.21 & 1.94 & .41 & .55 & .41 & & & & & \\
\hline$u$ & 3.47 & 1.78 & .39 & .59 & .44 & .62 & & & & \\
\hline $\log _{2}\left(t_{v}\right)$ & 2.96 & 0.75 & .24 & .19 & .14 & .18 & .16 & & & \\
\hline $\log _{2}\left(t_{w}\right)$ & 3.09 & 0.75 & .28 & .14 & .21 & .17 & .19 & .43 & & \\
\hline $\log _{2}\left(t_{d}\right)$ & 2.58 & 0.69 & .27 & .14 & .12 & .14 & .17 & .45 & .43 & \\
\hline $\log _{2}\left(t_{u}\right)$ & 2.49 & 0.71 & .27 & .18 & .14 & .16 & .16 & .41 & .43 & .47 \\
\hline
\end{tabular}

Note. Computed across $N=100$ participants and $k=12$ decision tasks. All correlations significant at $p$ $<.00005$

Conflict: Nomological validity. To the degree that our hypotheses are supported, the composite measure of conflict is related to tradeoff size and differential attribute importance in a theoretically meaningful way, attesting to its nomological validity. We further assess its nomological validity with respect to two behavioral criteria: choice probability and choice equiprobability.

For choice equiprobability, there is a positive relation between the degree to which the options are equally likely to be chosen and manifestations of conflict, for example, decision time and lack of confidence in the correctness of the decision reached (Festinger, 1943a, 1943b). This relation was already predicted by Cartwright and Festinger's (1943) quantitative theory of decision. For choice probability, there is a negative relation between the likelihood with which a particular option is chosen and manifestations of conflict, for example, decision time (Jamieson \& Petrusic, 1977; Petrusic \& Jamieson, 1978). Both relations are predicted by decision field theory.

To examine these relations, we determined, for each unique option pair in the design, the average level of conflict, the proportions of participants choosing option $x$ and option $y$, and the average levels of conflict associated with each choice. There were 60 unique option pairs: In each of 12 decision domains, two pairs implied a small tradeoff, two pairs implied an intermediate

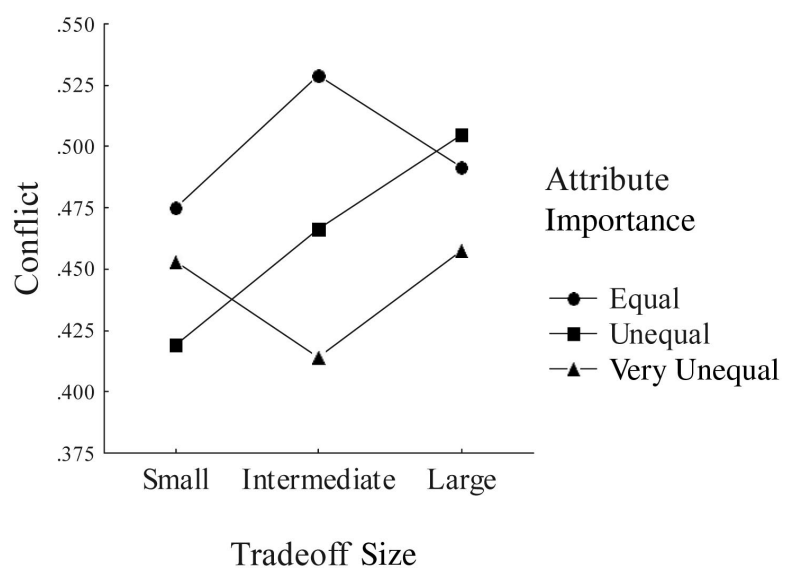

Figure 6. The observed relation between tradeoff size and conflict, as moderated by differential attribute importance. Conflict is in principal component scores, linearly transformed to a scale from 0 to 1 . tradeoff, and one pair implied a large tradeoff (see Figure 3). Two option pairs were discarded, because option $x$ was chosen by all participants presented with those pairs. For each of the 58 remaining option pairs, a measure of choice equiprobabiliy was obtained by applying an entropy measure to the choice proportions: Letting $P$ be the proportion of participants who chose a particular option, $E(P)=-\left[P \log _{2}(P)+(1-P) \log _{2}(1-P)\right]$, where $0<P<1$.

Both predicted relations emerged. First, across the 58 option pairs, the correlation between $E(P)$ and the average level of conflict was positive, $r=.36$, and significant, $t(56)=2.87, p=.00$, confirming that choice equiprobability increases with conflict. Second, across the 58 options pairs and the options within each pair, the correlation between $P$ and the average level of conflict associated with a particular choice was negative, $r=-.42$, and significant, $t(114)=-4.89, p=.00$, confirming that conflict decreases with choice probability. Altogether, then, the composite measure of conflict is related to choice probability and choice equiprobability in a way that is consistent with existing theory and evidence, further attesting to its nomological validity.

\section{Discussion}

The results of Experiment 1 confirm two of the three hypotheses, as derived from the double-mediation model, about how conflict is related to tradeoff size and how this relation is moderated by differential attribute importance. There was an inverse $U$-shaped relation between tradeoff size and conflict when one attribute was about as important as the other and a positive relation between tradeoff size and conflict when one attribute was more important than the other. However, there was a $U$-shaped relation between tradeoff size and conflict when one attribute was much more important than the other, whereas it was hypothesized that the relation between tradeoff size and conflict would (almost) vanish in such a situation. This was actually the least controversial hypothesis, which makes it all the more surprising that it was disconfirmed.

The double-mediation model can technically accommodate this $\mathrm{U}$-shaped relation between tradeoff size and conflict if it assumes conflict seeking, rather than conflict aversion, for situations of very unequal attribute importance. However, such an assumption would be implausible not only in relation to everything we know about how people cope with conflict but also in relation to the result as 
such: Why would people seek conflict when it is "obvious" which course of action to take?

We suspect that, as differential attribute importance increases to high levels, and the preliminary conflict decreases to low levels, deliberation is increasingly driven by perceptual aspects of the decision task. The $U$-shaped relation between tradeoff size and conflict suggests that, in situations of very unequal attribute importance, choice is dictated by the preliminary impression unless tradeoffs are conspicuously small, in which case people are alarmed by the very weak arguments in favor of either decision, or conspicuously large, in which case they are alarmed by the very great sacrifices to be incurred in choosing one option instead of the other. In the former case, conflict is mediated only by argumentation whereas, in the latter case, it is mediated only by sacrifice. Thus, in situations of very unequal attribute importance, conflict generation is no longer a matter of "double mediation." Instead, conflict is mediated by the source that is activated by the perceptually salient tradeoff. This gives rise to the $\mathrm{U}$-shaped relation between tradeoff size and conflict: The greater the salience of a tradeoff, the greater the conflict that is aroused by it.

The above interpretation is consistent with Borgida and Howard-Pitney's (1983) research on persuasion, which suggests that a perceptually salient cue is more likely to persuade the receivers of a message when those receivers are less likely to cognitively elaborate on message content. In Borgida and HowardPitney's research, however, a perceptually salient cue favored one of two attitudinal positions on an issue and, therefore, facilitated the evaluation task. What is surprising about our result is that perceptually salient tradeoffs complicated an otherwise simple decision task: A tradeoff between two attributes of very unequal importance strongly favored one of two options, so that it should be "obvious" which option to choose, but it often was not. This underscores Tversky and Simonson's (1993) conclusion that people often attend to "irrelevant" aspects of a situation and thus err by "unnecessarily" complicating their task.

The double-mediation model generally predicts an inverse $U$-shaped relation between tradeoff size and conflict which, under the influence of other aspects of the decision situation, may change in an upward or a downward direction. In Experiment 1, we examined differential attribute importance, a situational aspect that would change the inverse $U$-shaped relation in an upward direction. The purpose of Experiment 2 was to extend our analysis to an aspect that would change the inverse $U$-shaped relation in a downward direction.

\section{Experiment 2}

The double-mediation model introduces argumentation as a mediator of the relation between tradeoff size and conflict: Argumentation in the sense of justifying any decision to oneself. In Experiment 2, we examine how the relation between tradeoff size and conflict is moderated by the prospect of having to justify one's decision to others, more briefly referred to as need for justification. ${ }^{10}$

The left panel of Figure 7A shows a situation where one attribute is about as important as the other and there is no need for justification. This is a replica of the situation shown in the left panel of Figure 2A.

What are the implications of having to justify one's decision to others? We suggest that need for justification will shift attention toward argumentation and away from sacrifice: People will become more concerned with finding a strong argument for choosing one option or the other but, in shifting their attention, they will become less concerned with the sacrifice to be incurred in making that choice. Thus, the double-mediation model predicts that need for justification will increase the preliminary conflict generated by concern about argumentation but decrease the preliminary conflict generated by concern about sacrifice. Furthermore, the doublemediation model predicts that the rise in preliminary conflict generated by concern about argumentation will accentuate the relation between tradeoff size and the conflict generated by that concern, because it increases the associated drive for focused deliberation. Finally, the double-mediation model predicts that the fall in preliminary conflict generated by concern about sacrifice will attenuate the relation between tradeoff size and the conflict generated by that concern, because it decreases the associated drive for focused deliberation.

The right panel of Figure 7A shows a situation in which one attribute is about as important as the other and there is a need for justification. The negative relation between tradeoff size and the conflict generated by concern about argumentation has been accentuated while the positive relation between tradeoff size and the conflict generated by concern about sacrifice has been attenuated. As a result, the relation between tradeoff size and final conflict has changed in a downward direction.

In sum, we arrive at the following hypothesis about how the relation between tradeoff size and conflict is moderated by need for justification:

H2. Need for justification will change the relation between tradeoff size and conflict in a downward direction.

As can be seen in Figure 7B, need for justification has no clear overall effect on final conflict in this example: The final conflict aroused by smaller tradeoffs is greater when there is a need for justification, whereas the final conflict aroused by larger tradeoffs is greater when there is no such need.

Evidence from research on accountability suggests that, when people know the views that are held by the audience for their justifications, they tend to simply conform to those views; but, when they lack that knowledge, they tend to balance the pros and cons of different views that may be held by the audience (Tetlock, 1983; Tetlock, Skitka, \& Boettger, 1989). The above analysis of how need for justification moderates the relation between tradeoff size and conflict assumes that people do not know the preferences that are held by the audience of their justifications. To prevent our participants from knowing or thinking that they know those preferences, the identity of the audience was obscured (see "Participants and Procedure") and thus situations eliciting strong or socially desirable preferences were avoided.

For instance, the mileage of a car was much more important to the participants in Experiment 1 than its acceleration. Even when the audience and their preferences are unknown, the mere strength

\footnotetext{
${ }^{10}$ Here, justifying one's decision to others means explaining to others why one chose one option instead of the other. Elsewhere, in the literature on accountability, justifying one's decision to others means not only explaining the decision to others but also defending it against criticisms from others (Tetlock, 1983). There is evidence suggesting that the effect of expecting to explain one's decision is similar to the effect of expecting to explain and defend it (Simonson \& Nye, 1992, Study 4).
} 

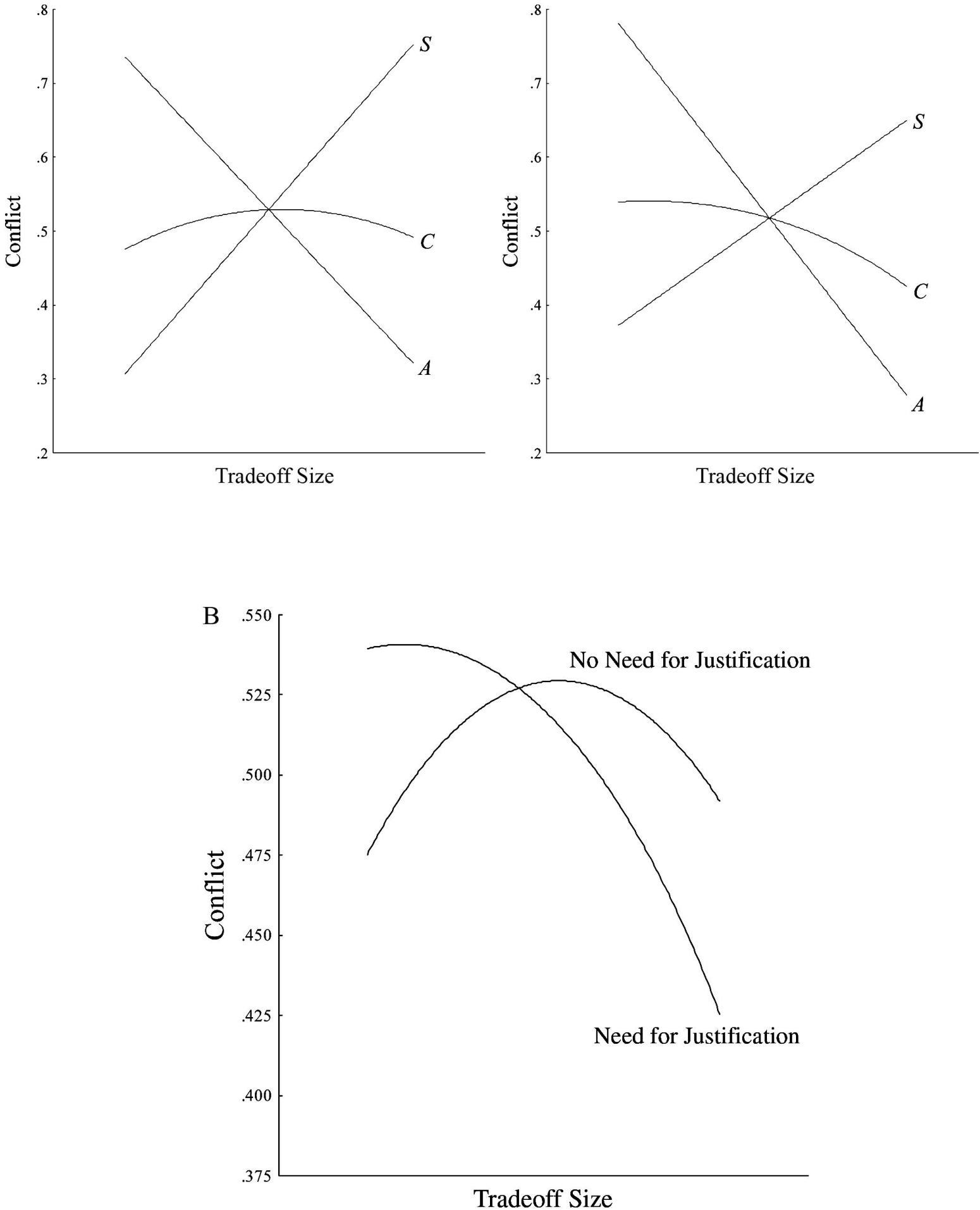

Figure 7. The double-mediation model. (A) The predicted relation between tradeoff size and conflict, as moderated by need for justification in a situation of equal attribute importance. (B) Summary of the relations between tradeoff size and final conflict. 
of preference for a car that is less costly in fuel consumption may increase people's tendency to overestimate the degree to which their preference is shared by others (false consensus). In addition, the social desirability of a preference for a car that is less wasteful of energy may by itself increase people's tendency to simply conform to that preference. Thus, to prevent our participants from second-guessing the preferences of the audience, situations eliciting strong or socially desirable preferences were avoided.

\section{Method}

Design and stimuli. Each participant completed two sets of three decision tasks in which, according to pretest results, one attribute was about as important as the other. The tasks in one set were taken from Experiment 1, but the attribute amounts were adjusted in accordance with new pretest results on perceived tradeoff sizes; the tasks in the other set were developed specifically for Experiment 2. The presentation order of the two sets was counterbalanced across participants. The set presented first was completed without justifications; the set presented last was completed with justifications required.

For each set of decision tasks, each participant completed one task in which the difference between the options along each attribute was perceived to be small and insignificant (small tradeoff), one task in which the difference between the options along each attribute was perceived to be large and significant (large tradeoff), and one task in which the perceived difference between the options along each attribute fell between these two extremes (intermediate tradeoff). So that any particular decision task would not always be associated with any particular tradeoff size, the association of tradeoff sizes with decision tasks was counterbalanced across six groups of participants.

Independently of tradeoff size, the option with respect to which tradeoffs were small, intermediate, or large was manipulated between participants, in that tradeoff size varied with respect to the option that was superior along one attribute for one half of the participants and with respect to the option that was superior along the other attribute for the other half (see Figure 3). As in Experiment 1, this control variable had no reliable effects on conflict and will not be discussed further.

As shown in Figure 3, the options composing the dyadic choice sets were located along a straight line, either in linearly spaced attribute amounts or, where pretest results revealed a greater sensitivity to differences near the worst end of one attribute than to differences near the best end of the other, in logarithmically spaced attribute amounts (up to slight deviations from the straight line where attribute amounts were rounded). Table 3 provides a sample of the stimulus material used in Experiment 2.

Participants and procedure. A total of 204 psychology students participated in the experiment and were paid $€ 7.50$. Experimental sessions were run by computer with at most 20 participants at a time. The procedure was the same as in Experiment 1, except for the changes required by the manipulation of need for justification. Specifically, the experiment consisted of a first part in which decisions were made without justifications and a second part in which decisions were made with justifications required. In the second part, the participants typed in the justification for a decision after having completed the decision task and accompanying rating tasks.

The participants were informed that the experiment consisted of two parts and that, in each part, they would complete several decision tasks, each accompanied by several rating tasks. They were given a description of

Table 3

Sample of the Stimulus Material Used in Experiment 2

\begin{tabular}{|c|c|c|c|c|c|c|}
\hline \multirow[b]{2}{*}{ Domain and attributes $^{b}$} & \multicolumn{6}{|c|}{ Dyadic choice sets $\{x, y\}^{\mathrm{a}}$} \\
\hline & $x$ & $y_{S}$ & $y_{\mathrm{I}}$ & $x_{\mathrm{I}}$ & $x_{S}$ & $y$ \\
\hline \multicolumn{7}{|l|}{ Digital cameras } \\
\hline \multirow[t]{5}{*}{1 Digital zoom } & $2 X$ & $4 X$ & & & & \\
\hline & $2 X$ & & $8 X$ & & & \\
\hline & & & & & & $16 \mathrm{X}$ \\
\hline & & & & $8 \times$ & & $16 \mathrm{X}$ \\
\hline & & & & & $12 X$ & $16 \mathrm{X}$ \\
\hline \multirow[t]{5}{*}{2 Storage capacity $(\mathrm{Mb})$} & 128 & 100 & & & & \\
\hline & 128 & & 70 & & & \\
\hline & 128 & & & & & 16 \\
\hline & & & & 67 & & 16 \\
\hline & & & & & 32 & 16 \\
\hline \multicolumn{7}{|l|}{ Printers } \\
\hline \multirow{5}{*}{1 Cost of color printing (cents) } & $71 / 2$ & 9 & & & & \\
\hline & $71 / 2$ & & 12 & & & \\
\hline & $71 / 2$ & & & & & 27 \\
\hline & & & & 17 & & 27 \\
\hline & & & & & 24 & 27 \\
\hline \multirow[t]{5}{*}{2 Speed of color printing (ppm) } & $31 / 2$ & 5 & & & & \\
\hline & $31 / 2$ & & 7 & & & \\
\hline & $31 / 2$ & & & & & 20 \\
\hline & & & & 11 & & 20 \\
\hline & & & & & 17 & 20 \\
\hline
\end{tabular}

Note. Complete listings of the stimulus material can be obtained from Scholten and Sherman (2004).

${ }^{\mathrm{a}}$ Small tradeoffs are implied by $\left\{x, y_{S}\right\}$ and $\left\{x_{S}, y\right\}$. Intermediate tradeoffs are implied by $\left\{x, y_{\mathrm{I}}\right\}$ and $\left\{x_{\mathrm{I}}, y\right\}$. A large tradeoff is implied by $\{x, y\}$.

${ }^{\mathrm{b}}$ As reported in the Results section, the attributes were of equal importance in only three decision tasks. Against our intentions, the attributes were of unequal importance in the other three decision tasks. The table contains an example from the situations in which Attribute 1 was about as important as Attribute 2 (digital cameras) and an example from the situations in which Attribute 1 was more important than Attribute 2 (printers), respectively. 
Table 4

Experiment 2: Means, Standard Deviations, and Correlations for Conflict Measures and Rating Times

\begin{tabular}{|c|c|c|c|c|c|c|c|c|c|c|}
\hline Variable & $M$ & $S D$ & $\log _{2}(t)$ & $E(v)$ & $E(w)$ & $d$ & $u$ & $\log _{2}\left(t_{v}\right)$ & $\log _{2}\left(t_{w}\right)$ & $\log _{2}\left(t_{d}\right)$ \\
\hline $\log _{2}(t)$ & 4.49 & 0.86 & & & & & & & & \\
\hline$E(v)$ & .83 & .16 & .30 & & & & & & & \\
\hline$E(w)$ & .79 & .17 & .28 & .62 & & & & & & \\
\hline$d$ & 4.19 & 1.97 & .39 & .59 & .44 & & & & & \\
\hline$u$ & 3.44 & 1.79 & .30 & .53 & .39 & .55 & & & & \\
\hline $\log _{2}\left(t_{v}\right)$ & 3.09 & 0.76 & .22 & .13 & .09 & .13 & .10 & & & \\
\hline $\log _{2}\left(t_{w}\right)$ & 3.23 & 0.76 & .32 & .13 & .14 & .11 & .06 & .34 & & \\
\hline $\log _{2}\left(t_{d}\right)$ & 2.67 & 0.71 & .21 & .12 & .11 & .09 & .06 & .42 & .41 & \\
\hline $\log _{2}\left(t_{u}\right)$ & 2.65 & 0.75 & .18 & .12 & .10 & .07 & .07 & .39 & .35 & .42 \\
\hline
\end{tabular}

Note. Computed across $N=204$ participants and $k=6$ decision tasks. All correlations significant at $p<.05$.

these tasks as well as instructions and rehearsal trials for keyboard handling. Before the session began, they were asked to provide thoughtful and truthful responses, relying on their personal tastes and judgments.

On concluding the first part, the participants learned that, in the second part, they would have to justify their decisions, meaning that they would have to explain why they had chosen one option instead of the other. They further learned that the justifications given by the participants in the experiment would be evaluated by an external committee and that a prize would be awarded to the participant whose justifications received the best evaluation from the committee. The participants were told that, if they turned out to be the winner, they would receive either $€ 50$ in cash or $€ 100$ in vouchers for the college bookshop and snack bar, whichever they preferred. They were asked to indicate which prize they would prefer to receive if they turned out to be the winner. The participants were further told that their responses would be treated with full confidentiality, but that the winner of the prize would have to be identified and notified. They were asked to type in their student number for this purpose only. The participants were then reminded that justifying their decisions meant explaining why they had chosen one option instead of the other, but this time it was added that merely referring to one attribute as being more important than the other would be classified as an incomplete justification by the evaluating committee. They were further reminded, before the session continued, to provide thoughtful and truthful responses, relying on their personal tastes and judgments.

\section{Results}

Check on equal attribute importance. Although the decision tasks were supposed to represent equal attribute importance, no pair of attributes is exactly equal in importance (see Footnote 9). As in Experiment 1, therefore, attribute 1 is designated as the more important attribute while attribute 2 is designated as the less important attribute.

A check on equal attribute importance was conducted by inspecting the relative frequency with which participants chose option $x$ (the option that was superior along attribute 1 ) when there was no need for justification. By the standards of Experiment 1, the attributes were about equally important: The probability of choosing option $x$ did not differ between the equal-importance condition of Experiment $1(.59)$ and the no-justification condition of Experiment 2 (.59), $z=-0.13, p=.90$. However, in Experiment 2 , the probability of choosing option $x$ was, against our intentions, systematically lower in one set of decision tasks (.53) than in the other $(.65), z=-2.86, p=.00 .{ }^{11}$ Thus, where appropriate, we separate these two sets, comprising three decision tasks each and representing situations of equal and unequal attribute importance, respectively.
Test of hypothesis: Dependent variable. Table 4 provides the means, standard deviations, and correlations for all measures taken in Experiment 2. We conducted a principal component analysis on the five conflict measures across the 204 participants and the 6 decision tasks. The first component had an eigenvalue of $\lambda=2.79$, thus accounting for $55.73 \%$ of the total variance. No other eigenvalues exceeded the unit threshold. The reliability of the unweighted composite of standardized conflict measures was $\alpha=$ .80. To improve reliability, we constructed a weighted composite measure of conflict, $\hat{C}$, by deriving the (standardized) scores on the first component and then linearly transforming these scores to a scale from 0 to 1 .

Test of hypothesis: Independent variables. The hypothesis that need for justification would change the relation between tradeoff size and conflict in a downward direction was tested with a linear regression analysis, run across participants and decision tasks. The analysis included 1 contrast between the 2 levels of need for justification, 1 contrast between the 2 levels of differential attribute importance, 4 orthogonal difference contrasts between the 3 replications of the respective levels of differential attribute importance (capturing the overall differences in conflict between replications), 2 orthogonal polynomial contrasts (linear and quadratic) between the 3 levels of tradeoff size, 1 contrast between the 2 levels of the control variable (the option with respect to which tradeoff size was varied), and 18 orthogonal contrasts capturing the interactions between the independent variables (need for justification, differential attribute importance, tradeoff size, and the control variable). On the basis of $H 2$, we expected that need for justification would interact with the linear trend in the relation between tradeoff size and conflict. More specifically, we expected that this interaction would have a negative coefficient.

To check whether the predicted interaction was robust across decision tasks, the regression analysis also included 4 contrasts capturing the variability of the interaction across the 3 replications of the respective levels of differential attribute importance which, in combination with the contrast capturing the variability of the interaction across the 2 levels of differential attribute importance ( 1 of the 18 interaction terms mentioned earlier), indicate the lack of robustness of the interaction across decision tasks. We evaluated

\footnotetext{
${ }^{11}$ A Newman-Keuls test had revealed significant differences in the probability of choosing option $x$ between decision tasks from different sets but not among the decision tasks from the same set.
} 
whether exclusion of these 5 contrasts, capturing the interaction among need for justification, linear trend, and decision task, yielded a reliable and meaningful decrease in goodness-of-fit $\left(R^{2}\right)$ of the regression model.

Test of hypothesis: Results. Figure 8 shows how the relation between tradeoff size and conflict is moderated by need for justification and differential attribute importance. Need for justification interacted significantly with the linear contrast between the levels of tradeoff size, $t(1192)=-3.11, p=.00$ : Consistent with $\mathrm{H} 2$, need for justification changed the relation between tradeoff size and conflict in a downward direction. More specifically, a positive relation changed into an inverse $U$-shaped relation (see top panel of Figure 8). Excluding the interaction between need for justification, linear trend, and decision task yielded a decrease in goodnessof-fit that was very small, $.145-.150=-.005$, and insignificant, $F(26,31)=1.53, p=.13$. Thus, the predicted effect was robust across decision tasks.

Three other results emerged, all consistent with the evidence from Experiment 1. First, the contrast between the levels of differential attribute importance was significant, $t(1192)=-9.85, p=.00$ : Conflict decreased with differential attribute importance. Second, the
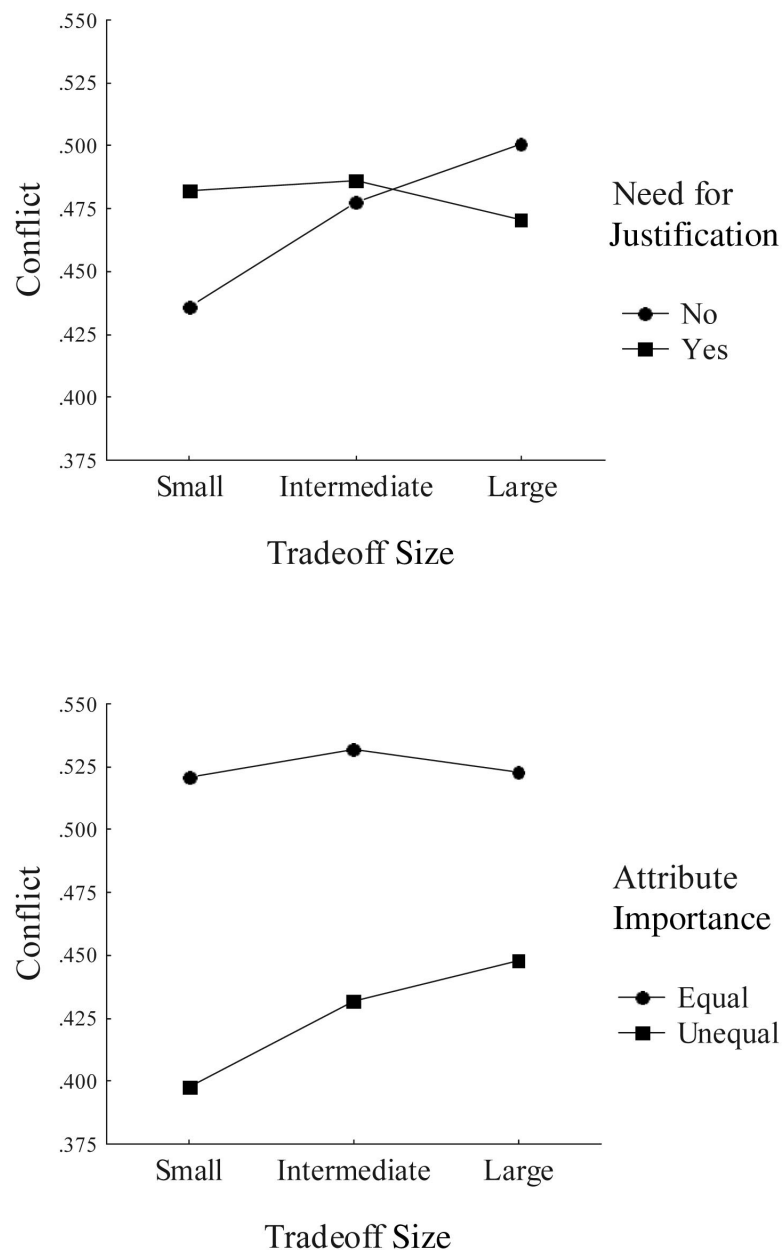

Figure 8. The observed relation between tradeoff size and conflict, as moderated by need for justification (top panel) and differential attribute importance (bottom panel). Conflict is in principal component scores, linearly transformed to a scale from 0 to 1 . linear contrast between the levels of tradeoff size was significant, $t(1192)=2.15, p=.03$ : Conflict generally increased with tradeoff size. Third, differential attribute importance interacted significantly with the linear contrast between the levels of tradeoff size, $t(1192)=$ $1.94, p=.05$ : Differential attribute importance changed the relation between tradeoff size and conflict in an upward direction. More specifically, an inverse $U$-shaped relation changed into a positive relation (see bottom panel of Figure 8).

Conflict: Convergent and discriminant validity. Convergent validity was assessed in terms of the correlation between decision time and a composite of the rating measures of conflict (in principal component scores), which was moderately high, $r=.40$, and significant, $t(1222)=15.05, p=.00$. There was no inverse $U$-shaped relation between conflict and decision time but rather a positive, slightly accelerating one. We conducted a linear and a polynomial (quadratic) regression analysis with decision time as dependent variable and the composite of the rating measures as independent variable. The quadratic model yielded an increase in goodness-of-fit that was significant, $F(1,1221)=12.43, p=.00$, but small, $.17-.16=.01$. Discriminant validity was assessed in terms of the difference between, on the one hand, the correlation between decision time and the composite of the rating measures and, on the other hand, the correlation between decision time and a composite of the rating times (in principal component scores). This difference was positive, $.40-.31=.08$, and significant, $t(1221)=2.50, p=.01$ (by Hotelling's $t$-statistic).

Conflict: Nomological validity. We assessed whether the composite conflict measure was negatively related to choice probability and positively related to choice equiprobability. Thus, we determined, for each unique option pair in the design, the average level of conflict, the proportions of participants choosing option $x$ and option $y$, and the average levels of conflict associated with each choice. There were 30 unique option pairs: In each of 6 decision domains, 2 pairs implied a small tradeoff, 2 pairs implied an intermediate tradeoff, and 1 pair implied a large tradeoff (see Figure 3). For each option pair, a measure of choice equiprobabiliy was obtained by applying an entropy measure, $E(P)$, to the choice proportions, $P$.

Across the 30 option pairs, the correlation between $E(P)$ and the average level of conflict was positive, $r=.52$, and significant, $t(28)=3.19, p=.00$, confirming that choice equiprobability increases with conflict. However, across the 30 options pairs and the options within each pair, the correlation between $P$ and the average level of conflict associated with a particular choice was negative, $r=-.18$, and approached significance, $t(58)=-1.37$, $p=.09$, confirming that conflict decreases with choice probability.

Justifications: Protocol analysis. The protocols were analyzed by three independent judges. The contents of the justifications were covered by six categories: (a) conflict; (b) the relative importance of the attributes; (c) the size of the tradeoff between the attributes; (d) desire or need for the product; (e) familiarity with the product; and (f) financial, physical, psychic, or temporal constraints on the acquisition or utilization of the product. The composite interjudge reliability coefficient was .84 . Disagreements were resolved by discussion. The proportions of references to the categories were $.06, .54, .16, .14, .13$, and .05 , respectively. Thus, the majority of references were made to the relative importance of the attributes. The correlations between references to the categories hovered around zero, except for those between references to relative attribute importance and references to the other categories, which were significantly below zero $(r=-.30$, averaged across 
categories). Thus, references to relative attribute importance tended to inhibit references to all other categories.

For each category of substantive interest (conflict, the relative importance of the attributes, and the size of the tradeoff between the attributes), we ran a nonlinear regression analysis (LOGIT) across participants and decision tasks. The dependent variable was reference to the category. The independent variables were those included in the linear regression analysis testing $H 2$, this time also including conflict but excluding need for justification and its interactions with the other variables.

Conflict stimulated references to conflict, $\chi^{2}(1)=69.78, p=$ .00 , which further attests to the convergent validity of the composite conflict measure, and inhibited references to two principal causes of conflict: (a) the relative importance of the attributes, $\chi^{2}(1)=5.31, p=.02$, and (b) the size of the tradeoff between the attributes, $\chi^{2}(1)=3.81, p=.05$. Tradeoff size had two effects on references to tradeoff size. First, small or large tradeoffs stimulated more such references than tradeoffs of intermediate size, $.17-$ $.08=.09, \chi^{2}(1)=9.06, p=.00$. Second, small tradeoffs stimulated more such references than large tradeoffs, $.21-.13=.09$, $\chi^{2}(1)=5.39, p=.02$. The former result suggests that people are alerted by conspicuously small or large tradeoffs even when perceptual salience of tradeoffs has no apparent consequences for conflict generation (see the "Discussion" of Experiment 1). The latter result suggests that, when there is a need for justification, people are alerted more by small tradeoffs than by large ones, which is entirely consistent with the theoretical analysis in the introduction to this experiment.

\section{Discussion}

The results of Experiment 2 confirm the hypothesis, as derived from the double-mediation model, that need for justification changes the relation between tradeoff size and conflict in a downward direction. Although our intention was to test this hypothesis in a situation of equal attribute importance, only half of the decision tasks represented such a situation. The other half represented a situation of unequal attribute importance. Accordingly, tradeoff size had a positive relation with conflict, which became an inverse $\mathrm{U}$-shaped relation when there was a need for justification (top panel of Figure 8), in contrast with our earlier example, in which tradeoff size had an inverse $U$-shaped relation with conflict, which became a negative relation when there was a need for justification (Figure 7B). However, the double-mediation model can accurately account for the results of our experiment.

Figure 9A shows how need for justification moderates the relation between tradeoff size and conflict in a situation of unequal attribute importance. In comparison to Figure $7 \mathrm{~A}$, the relations between tradeoff size and the conflict generated by concern about sacrifice and concern about argumentation are attenuated, because of an overall decline in preliminary conflict levels and associated drives for focused deliberation. Specifically, the situation in which is no need for justification, shown in the left panel of Figure 9A, is similar to the situation shown in the center panel of Figure 2A: The negative relation between tradeoff size and the conflict generated by concern about argumentation is attenuated to a greater degree than the positive relation between tradeoff size and the conflict generated by concern about sacrifice, giving rise to a positive relation between tradeoff size and final conflict. The right panel of Figure 9A shows a situation in which there is a need for justification. In comparison to the left panel, the relation between tradeoff size and the conflict generated by concern about argumentation is accentuated but the relation between tradeoff size and the conflict generated by sacrifice is attenuated, as theorized, changing the positive relation between tradeoff size and final conflict into an inverse $U$-shaped relation (see also the summary in Figure 9B).

Given that the attributes were of equal importance in half of the decision tasks but of unequal importance in the other half, we had an additional opportunity to test the principal hypothesis of Experiment 1 , according to which a change from equal to unequal attribute importance will change the relation between tradeoff size and conflict in an upward direction, more specifically, change an inverse $U$-shaped relation into a (more) positive relation. The results of Experiment 2 also confirm this hypothesis (bottom panel of Figure 8).

The intended effect of the need-for-justification manipulation was that our participants would shift their attention toward argumentation and away from sacrifice. For reasons given in the introduction to this experiment, the audience of their justifications had to remain unknown. To ensure that the audience would nonetheless be relevant to them (why else bother justifying their decisions to others?), a prize was awarded to the participant whose justifications were received most favorably by the audience. Thus, the prize was integral to the purpose of the manipulation. For those interested in the effects of need for justification per se, the prize may be a confounding factor, although we do not see how a prize per se (or, indeed, typing the justification per se) could have produced our results.

In our experimental design, the set of decision tasks presented first was completed without justifications, and the set presented last was completed with justifications. It would have been inappropriate to counterbalance the order of the need-for-justification conditions, because participants going through the reverse order would probably adopt a justification frame of mind when asked to justify their decisions but not abandon that frame of mind when no longer asked for justifications. Moreover, in our experimental design, any order effect probably countervails the moderating effect of need for justification, because experience with earlier decision tasks, particularly those in which attributes are of unequal importance, may be applied to later ones, so that, according to the double-mediation model, the relation between tradeoff size and conflict should become more positive. The order effect can actually be tested within the experimental conditions (need for justification and no such need). We therefore ran an additional regression analysis that included, among the independent variables, the ordinal position of a decision task (randomized across participants) and its interactions with the other variables. ${ }^{12}$ As expected, the relation between tradeoff size and conflict was more positive when a decision task appeared later than when it appeared earlier, $t(1$, $1184)=2.42, p=.02$, and this order effect was specific to decision tasks in which the attributes were of unequal importance, $t(1,1184)=2.22, p=.03$. Thus, if the ordinal position of those decision tasks had an analogous effect between the experimental conditions, our hypothesis about the moderating effect of need for justification was confirmed despite a countervailing order effect.

\footnotetext{
${ }^{12}$ We excluded the control variable indicating the option with respect to which tradeoff size varied and its interactions with the other variables, which all had negligible effects in the original analysis.
} 
A

No Need for Justification

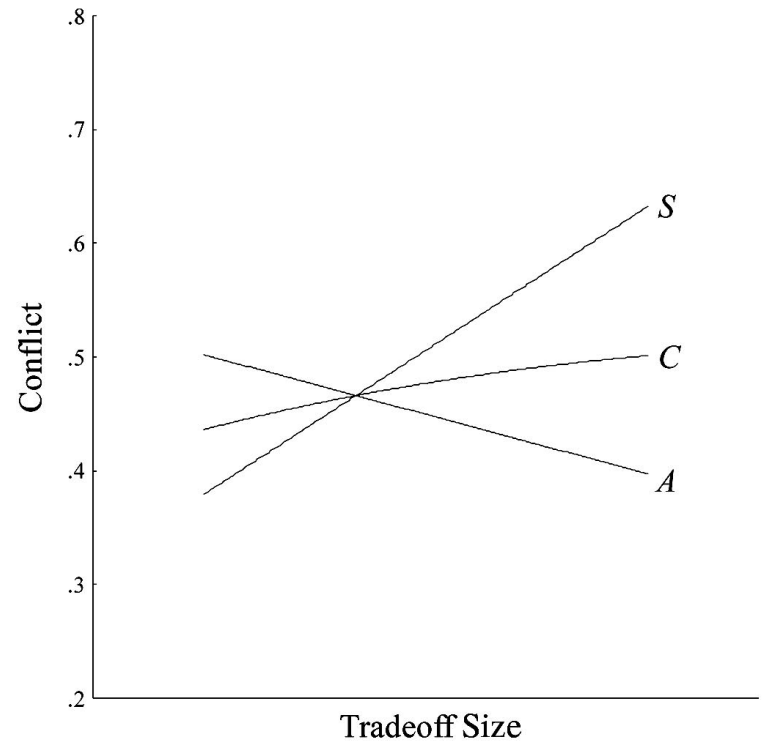

Need for Justification

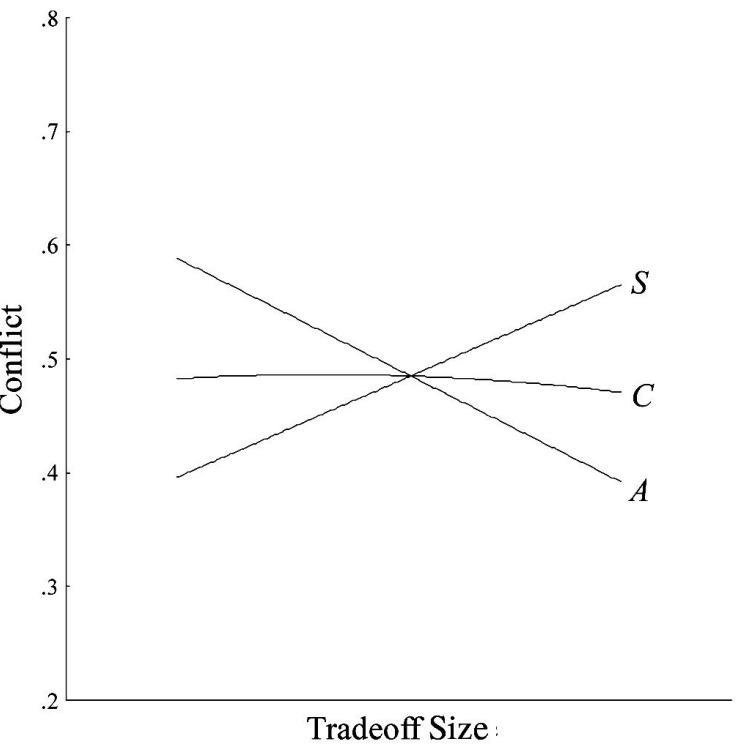

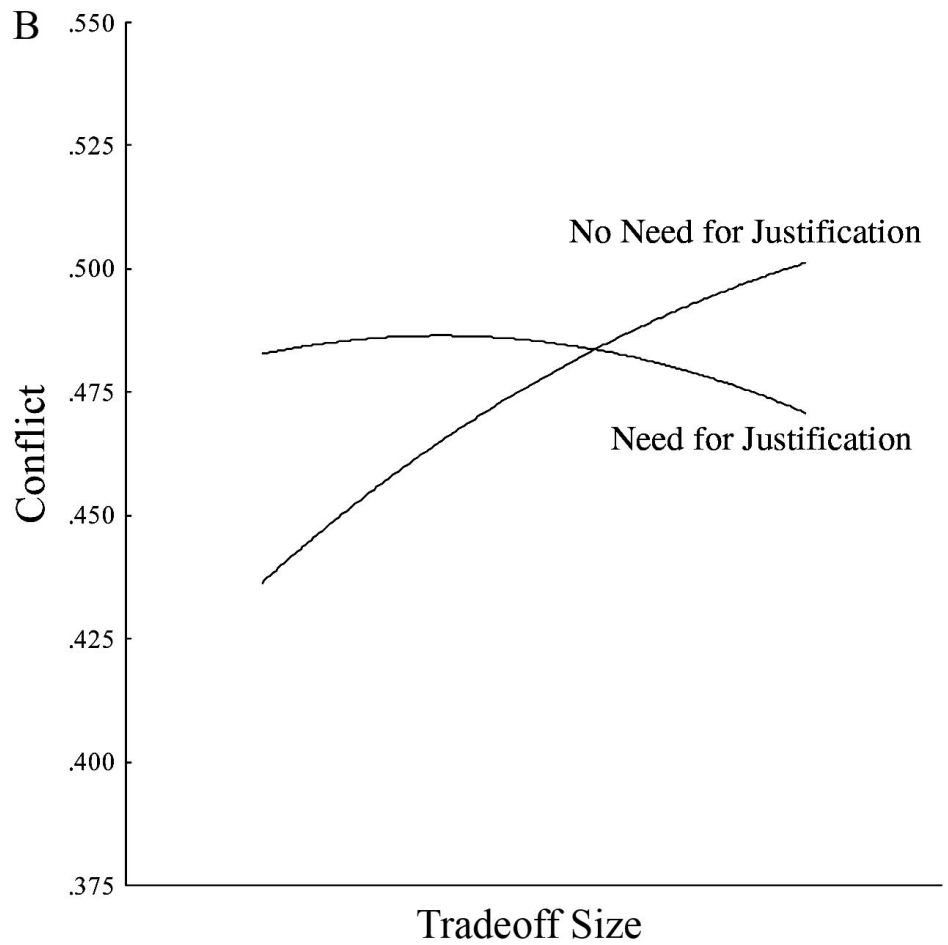

Figure 9. The double-mediation model. (A) The predicted relation between tradeoff size and conflict, as moderated by need for justification in a situation of unequal attribute importance. (B) Summary of the relations between tradeoff size and final conflict. 


\section{General Discussion}

Real choices involve tradeoffs and tradeoffs induce conflict. The double-mediation model describes how tradeoffs induce conflict and, on this basis, how conflict is related to the size of tradeoffs. Our model suggests that this relation can take on many forms and that the specific shape depends on the decision situation.

The double-mediation model introduces three ingredients to the explanation of decisional conflict: First, the existence of two sources of conflict in preference formation (concern about sacrifice and concern about argumentation); second, the prevalence of the source from which the least conflict is elicited (conflict aversion); and third, the capacity of the preliminary conflict elicited from either source as a drive for focused deliberation (orientation reaction). The first two ingredients give rise to an inverse $U$-shaped relation between tradeoff size and final conflict. The third ingredient gives rise to situational dependence, in that the decision situation may change the inverse $U$-shaped relation into a (more) positive or (more) negative relation.

Our experimental results generally support the predictions of the double-mediation model, both qualitatively and quantitatively. However, future investigation may extend the analysis to decision domains other than consumer choice. Obvious candidates would be risky choice and intertemporal choice, both prominent objects of study in the field of behavioral decision making. The analysis may further be extended to other aspects of the decision situation. For instance, consumer products may be differentiated by discrete features rather than continuous attributes (e.g., Dhar \& Sherman, 1996), the choice options may be presented sequentially rather than simultaneously (e.g., Dhar, 1996), and the choice set may include a no-choice option (free choice) rather than exclude it (forced choice; e.g., Dhar \& Simonson, 2003).

As we described in the beginning of this article, the idea of a double-mediation model originated from the contradiction between what most theories of decision making suggest about how conflict is related to tradeoff size and what many people believe about this relation. On detecting this contradiction, we asked ourselves, "Which view is correct?" Because either one seemed intuitively plausible to us, our answer was, "Both." Subsequent thinking about the implications of a double-mediation process for the relation between tradeoff size and final conflict was greatly inspired by McGuire's (1968) information-processing model of persuasion. Because the information-processing model played a vital role in the evolution of the double-mediation model, we discuss below the commonalities and differences between these two formulations.

\section{Comparison With the Information-Processing Model of Persuasion}

The double-mediation model, in which the conflict aroused by a tradeoff is mediated by sacrifice and argumentation, and a rudimentary version of the information-processing model, in which persuasion by a message is mediated by reception and yielding, can be summarized in exactly the same way: (a) The relation between an independent variable and a dependent variable is mediated by two factors; the mediating effect of one factor is positive, whereas the mediating effect of the other factor is negative; an interaction of the opposite effects gives rise to an inverse $\mathrm{U}$-shaped relation between the independent variable and the dependent variable; and third variables may change the relative magnitude of the mediating effects, thus changing the inverse $\mathrm{U}$-shaped relation into a (more) positive or (more) negative relation. Despite these similarities, however, the models are very different, both conceptually and mathematically. Below, we discuss three conceptual differences.

Justification for opposite effects. McGuire's (1968) two-factor model assumes that an independent variable tends to affect reception and yielding in opposite directions. However, the justification for assuming opposite effects is not very convincing: As adapted to the two-factor model, "people and the world are so structured that quite frequently a variable which affects one [factor] positively tends to affect [the other factor] negatively" (McGuire, 1978, p. 170). Actually, assuming, rather than simply allowing for, opposite effects seems to expose the information-processing model to needless risk of disconfirmation (Scholten, 1996): The assumption may hold in some situations (e.g., receiver intelligence helps reception but hurts yielding; see McGuire, 1968, 1969) but not in others (e.g., source credibility helps yielding but does not affect reception; see McGuire, 1969). The double-mediation model also assumes that an independent variable affects one mediating factor in a positive direction and another mediating factor in a negative direction. However, it provides a simple and compelling justification for assuming opposite effects: Larger tradeoffs mean greater sacrifices, thus leading to a greater conflict, but also stronger arguments, thus leading to less conflict.

Justification for interacting effects. In the informationprocessing model, reception and yielding are two successive stages of the persuasion process. The interaction of their mediating effects has a logical justification: It is not possible to reach the yielding stage without having reached the reception stage first. In the double-mediation model, concern about sacrifice and concern about argumentation are two sources of conflict that operate simultaneously in the process of preference formation. The interaction of their mediating effects has a psychological justification: Conflict is an unpleasant state with which people tend to cope along the route of least resistance, so that given two levels of conflict from two sources, the lower level of conflict, whatever its source, will contribute more to final conflict.

Attenuation of mediating effects. In the informationprocessing model, the mediating effect of either stage of the persuasion process (reception or yielding) may be attenuated in a downward direction, when a third variable makes it less likely that the stage will be reached, or in an upward direction, when a third variable makes it more likely that the stage will be reached. In general, the mediating effect of either stage of the persuasion process increases as the prior uncertainty of reaching that stage increases. In the double-mediation model, any attenuation of the mediating effect of either source of conflict (concern about sacrifice or concern about argumentation) is always in a downward direction, because the mediating effect is attenuated only when a third variable decreases the preliminary conflict elicited from that source and, thus, the drive for focused deliberation. In general, the mediating effect of either source of conflict increases as the preliminary conflict elicited from that source increases.

In sum, there are three conceptual differences between the double-mediation model of conflict generation and a model that greatly inspired its evolution, the information-processing model of persuasion. These three differences correspond to the three ingredients that the double-mediation model has introduced to the 
explanation of decisional conflict: Double mediation, conflict aversion, and orientation reaction.

Inverse $\mathrm{U}$-shaped relations abound in psychology, and they can be found in behavioral decision making as well. It is perhaps useful to compare the double-mediation model with Coombs' (1987; Coombs \& Avrunin, 1977) theory of single-peaked preference functions, because of its allusion to "the structure of conflict."

\section{Comparison With the Theory of Single-Peaked Preference Functions}

The double-mediation model generally predicts an inverse U-shaped relation between tradeoff size and conflict. We tested this prediction in situations where, as shown in Figure 3, a large tradeoff was implied by two extreme options while intermediate and small tradeoffs were implied by either extreme option and less extreme options. Coombs (1987; Coombs \& Avrunin, 1977) has specified four conditions that give rise to a single-peaked preference function in such situations, meaning that, as we move from one extreme end of the option set to the other, preference for the options first increases and then decreases. Below, we discuss this formulation, showing why, given the stimulus design in Figure 3, an inverse $U$-shaped relation between tradeoff size and conflict is incompatible with a single-peaked preference function.

A primary condition for single-peaked preference functions is that the preference be either increasing in attribute amounts ("more is better") or decreasing in attribute amounts ("less is better"). Three kinds of tradeoffs may be distinguished, all of which were included in the stimulus material for our experiments: Approachapproach conflicts, when more is better along both attributes, for example, jobs implying a tradeoff between contract duration and monthly wage, avoidance-avoidance conflicts, when less is better along both attributes, for example, a laser printer and a inkjet printer implying a tradeoff between cost per print and price, and approach-avoidance conflicts, when more is better along one attribute but less is better along the other, for example, cellular phones implying a tradeoff between talk time and weight.

Coombs specifies three more conditions for these kinds of tradeoffs so as to arrive at single-peaked preference functions, one addressing the valuation of attribute amounts (how attribute amounts are transformed into attribute values), another addressing the valuation of options (how attribute values are integrated into an overall value), and still another addressing the pattern of tradeoff rates implied by adjacent options (how the rates of exchange between attributes 1 and 2 evolve when moving from one extreme of the option set to the other). The details of these conditions are not important here.

What is important, however, is that, given the stimulus design in Figure 3 , an inverse $U$-shaped relation between tradeoff size and conflict is incompatible with a single-peaked preference function: The large tradeoff (between the two extreme options) and the small tradeoffs (between either one of the extreme options and the adjacent option) should have resulted in the smallest differences in overall value between the options and, thus, in the highest levels of conflict, while the intermediate tradeoffs should have resulted in the greatest differences in overall value between the options and, thus, in the lowest levels of conflict. In other words, there should have been a $U$-shaped relation between tradeoff size and conflict, the reverse of what we predicted and observed.

Although several conditions specified by Coombs may have been violated, we believe that the independent valuation of options is an inaccurate description of preference formation in the decision situations being considered (viz., simultaneous presentation of two options implying a tradeoff between two attributes). The double-mediation model suggests that, in such situations, preference formation involves direct comparisons between the attribute amounts delivered by the options, rather than comparisons between overall values of the options deriving from those attribute amounts. This different perspective on preference formation has different implications for conflict generation.

\section{Conclusion}

We have developed the double-mediation model as an autonomous model of conflict generation, drawing on the assumptions about preference formation from decision field theory. As mentioned earlier, decision field theory can be viewed as a theory of decision making, by predicting the probability that a particular decision will be made, and a theory of the conflict involved in decision making, by predicting the average time it will take to make that decision. Future investigation may determine whether and how decision field theory can accommodate the processes of conflict generation described by the double-mediation model. If it can, it would improve our understanding of choice and conflict.

\section{References}

Anderson, N. H. (1971). Integration theory and attitude change. Psychological Review, 78, 171-206.

Anderson, N. H. (1972). Looking for configurality in clinical judgment. Psychological Bulletin, 78, 93-102.

Berlyne, D. E. (1957a). Conflict and choice time. British Journal of Psychology, 48, 106-118.

Berlyne, D. E. (1957b). Uncertainty and conflict: A point of contact between information-theory and behavior-theory concepts. Psychological Review, 64, 329-339.

Berlyne, D. E. (1961). Conflict and the orientation reaction. Journal of Experimental Psychology, 62, 476-483.

Berlyne, D. E. (1962). Comments on Prokasy's reply. Psychological Reports, 11, 581-582.

Berlyne, D. E. (1966). Conflict and reaction time: Reply to Kiesler. Psychological Reports, 19, 413-414.

Bettman, J. R., Johnson, E. J., Luce, M. F., \& Payne, J. W. (1993) Correlation, conflict, and choice. Journal of Experimental Psychology: Learning, Memory, and Cognition, 19, 931-951.

Borgida, E., \& Howard-Pitney, B. (1983). Personal involvement and the robustness of perceptual salience effects. Journal of Personality and Social Psychology, 45, 560-570.

Busemeyer, J. R., \& Diederich, A. (2002). Survey of decision field theory. Mathematical Social Sciences, 43, 345-370.

Busemeyer, J. R., \& Townsend, J. T. (1993). Decision field theory: A dynamic-cognitive approach to decision-making in an uncertain environment. Psychological Review, 100, 432-459.

Campbell, D. T., \& Fiske, D. W. (1959). Convergent and discriminant validation by the multitrait-multimethod matrix. Psychological Bulletin, 56, 81-105.

Cartwright, D., \& Festinger, L. (1943). A quantitative theory of decision. Psychological Review, 50, 595-621.

Chatterjee, S., \& Heath, T. B. (1996). Conflict and loss aversion in multiattribute choice: The effects of trade-off size and reference dependence on decision difficulty. Organizational Behavior and Human Decision Processes, 67, 144-155.

Coombs, C. H. (1987). The structure of conflict. American Psychologist, $42,355-363$. 
Coombs, C. H., \& Avrunin, G. S. (1977). Single-peaked functions and the theory of preference. Psychological Review, 84, 216-230.

Dhar, R. (1996). The effect of decision strategy on deciding to defer choice. Journal of Behavioral Decision Making, 9, 265-281.

Dhar, R. (1997). Consumer preference for a no-choice option. Journal of Consumer Research, 24, 215-231.

Dhar, R., \& Nowlis, S. M. (1999). The effect of time pressure on consumer choice deferral. Journal of Consumer Research, 25, 369-384.

Dhar, R., \& Nowlis, S. M. (2004). To buy or not to buy: Response mode effects on consumer choice. Journal of Marketing Research, 41, 423-432.

Dhar, R., \& Sherman, S. J. (1996). The effect of common and unique features in consumer choice. Journal of Consumer Research, 23, 193-203.

Dhar, R., \& Simonson, I. (2003). The effect of forced choice on choice. Journal of Marketing Research, 40, 146-160.

Diederich, A. (1997). Dynamic stochastic models for decision making with time constraints. Journal of Mathematical Psychology, 41, 260-274.

Diederich, A. (2003). Decision making under conflict: Decision time as a measure of conflict strength. Psychonomic Bulletin and Review, 10, 167-176.

Festinger, L. (1943a). Studies of decision: I. Decision-time, relative frequency of judgment and subjective confidence as related to physical stimulus difference. Journal of Experimental Psychology, 32, 291-306.

Festinger, L. (1943b). Studies of decision: II. An empirical test of a quantitative theory of decision. Journal of Experimental Psychology, 32, 411-423.

Festinger, L. (1964). Conflict, decision, and dissonance. Stanford, CA: Stanford University Press.

Fischer, G. W., Jia, J., \& Luce, M. F. (2000a). Attribute conflict and preference uncertainty: The RandMAU model. Management Science, 46, 669-684.

Fischer, G. W., Luce, M. F., \& Jia, J. (2000b). Attribute conflict and preference uncertainty: Effects on judgment time and error. Management Science, 46, 88-103.

Fishburn, P. C. (1974). Lexicographic orders, utilities and decision rules: A survey. Management Science, 20, 1442-1471.

Goldstein, W. M. (1990). Judgments of relative importance in decision making: Global vs. local interpretations of subjective weight. Organizational Behavior and Human Decision Processes, 47, 313-336.

Jamieson, D. G., \& Petrusic, W. M. (1977). Preference and time to choose. Organizational Behavior and Human Performance, 19, 56-67.

Janis, I. L. (1959). Motivational factors in the resolution of decisional conflicts. In M. R. Jones (Ed.), Nebraska Symposium on Motivation (Vol. 8, pp. 198-231). Lincoln, NE: University of Nebraska Press.

Kahneman, D., \& Tversky, A. (1979). Prospect theory: An analysis of decision under risk. Econometrica, 47, 263-291.

Kiesler, C. A. (1966). Conflict and the number of choice alternatives. Psychological Reports, 18, 603-610.

Lindeman, R. H., Merenda, P. F., \& Gold, R. Z. (1980). Introduction to bivariate and multivariate analysis. Glenview, IL: Scott, Foresman.

Luce, M. F. (1998). Choosing to avoid: Coping with negatively emotionladen consumer decisions. Journal of Consumer Research, 24, 409-433.

Luce, M. F., Bettman, J. R., \& Payne, J. W. (1997). Choice processing in emotionally difficult decisions. Journal of Experimental Psychology: Learning, Memory, and Cognition, 23, 384-405.

Luce, M. F., Jia, J., \& Fischer, G. W. (2003). How much do you like it? Within-alternative conflict and subjective confidence in consumer judgments. Journal of Consumer Research, 30, 464-472.

McGuire, W. J. (1968). Personality and susceptibility to social influence. In E. F. Borgatta \& W. W. Lambert (Eds.), Handbook of personality theory and research (pp. 1130-1187). Chicago: Rand McNally.

McGuire, W. J. (1969). The nature of attitudes and attitude change. In G. Lindzey \& E. Aronson (Eds.), Handbook of social psychology (Vol. 3, pp. 136-314). Reading MA: Addison Wesley.

McGuire, W. J. (1978). An information-processing model of advertising effectiveness. In H. L. Davis \& A. H. Silk (Eds.), Behavioral and management sciences in marketing (pp. 156-180). New York: Ronald Press.

Nowlis, S. M. (1995). The effect of time pressure on the choice between brands that differ in quality, price, and product features. Marketing Letters, 6, 287-295.

Nowlis, S. M., \& Simonson, I. (1996). The effect of new product features on brand choice. Journal of Marketing Research, 33, 36-46.

Petrusic, W. M., \& Jamieson, D. G. (1978). Relation between probability of preferential choice and time to choose changes with practice. Journal of Experimental Psychology: Human Perception and Performance, 4, 471-482.

Russo, J. E., Meloy, M. G., \& Medvec, V. H. (1998). Predecisional distortion of product information. Journal of Marketing Research, 35, $438-452$.

Scholten, M. (1996). Lost and found: The information-processing model of advertising effectiveness. Journal of Business Research, 37, 97-104.

Scholten, M. (2002). Conflict-mediated choice. Organizational Behavior and Human Decision Processes, 88, 683-718.

Scholten, M., \& Sherman, S. J. (2004). Tradeoffs, conflict, and choice: The double-mediation model (Tech. Rep. No. 254 of the Cognitive Science Program at Indiana University). Retrieved April 7, 2006, from http:// www.cogs.indiana.edu/publications/index.html\#techreps.

Shafir, E., Simonson, I., \& Tversky, A. (1993). Reason-based choice. Cognition, 49, 11-36.

Shepard, R. N. (1964). On subjectively optimum selection among multiattribute alternatives. In M. W. Shelley, II \& G. L. Bryan (Eds.), Human judgments and optimality (pp. 257-281). New York: Wiley.

Shugan, S. M. (1980). The cost of thinking. Journal of Consumer Research, 7, 99-111.

Simonson, I. (1989). Choice based on reasons: The case of attraction and compromise effects. Journal of Consumer Research, 16, 158-174.

Simonson, I., \& Nye, P. (1992). The effect of accountability on susceptibility to decision errors. Organizational Behavior and Human Decision Processes, 51, 416-446.

Simonson, I., \& Tversky, A. (1992). Choice in context: Tradeoff contrast and extremeness aversion. Journal of Marketing Research, 29, 281-295.

Slovic, P. (1975). Choice between equally valued alternatives. Journal of Experimental Psychology: Human Perception and Performance, 1, $280-287$.

StatSoft. (2003). Statistica (Version 6) [Computer software]. Tulsa, OK: Author.

Tetlock, P. E. (1983). Accountability and complexity of thought. Journal of Personality and Social Psychology, 45, 74-83.

Tetlock, P. E., Skitka, L., \& Boettger, R. (1989). Social and cognitive strategies for coping with accountability: Conformity, complexity, and bolstering. Journal of Personality and Social Psychology, 57, 632-640.

Tversky, A. (1972). Elimination by aspects: A theory of choice. Psychological Review, 79, 281-299.

Tversky, A., \& Sattath, S. (1979). Preference trees. Psychological Review, $86,542-573$.

Tversky, A., Sattath, S., \& Slovic, P. (1988). Contingent weighting in judgment and choice. Psychological Review, 95, 371-384.

Tversky, A., \& Shafir, E. (1992). Choice under conflict: The dynamics of deferred decision. Psychological Science, 3, 358-361.

Tversky, A., \& Simonson, I. (1993). Context-dependent preferences. Management Science, 39, 1179-1189.

Tyebjee, T. T. (1979). Response time, conflict, and involvement in brand choice. Journal of Consumer Research, 6, 295-304.

Wood, W. (2000). Attitude change: Persuasion and social influence. Annual Review of Psychology, 51, 539-570.

Zakay, D. (1985). Post-decisional confidence and conflict experienced in a decision process. Acta Psychologica, 58, 75-80.

Zakay, D., \& Tsal, Y. (1993). The impact of using forced decision making strategies on post-decisional confidence. Journal of Behavioral Decision Making, 6, 53-68. 


\section{Appendix}

In this appendix, we provide a fully parametric formulation of the double-mediation model, estimate its parameters from our experimental results, and perform parametric tests of our hypotheses.

\section{Parametric Formulation of the Double-Mediation Model}

Final conflict, $C$, is a function of conflict generated by concern about sacrifice, $S$, and conflict generated by concern about argumentation, $A$. Applying Anderson's (1971) integration theory,

$$
C=(1-w) S+w A,
$$

where $0 \leq w \leq 1$ and, without loss of generality, conflict is expressed on a scale from 0 to 1 . In this formulation, $1-w$ is the relative weight of $S$, and $w$ is the relative weight of $A$. The relative weight $w$ can be written as a function of two absolute weights (see also Anderson, 1972):

$$
w=\frac{\Omega_{S}}{\Omega_{S}+\Omega_{A}} .
$$

where $\Omega_{S}, \Omega_{A} \geq 0$. Substituting Equation A2 into Equation A1,

$$
C=\frac{\Omega_{A}}{\Omega_{S}+\Omega_{A}} S+\frac{\Omega_{S}}{\Omega_{S}+\Omega_{A}} A .
$$

Given conflict aversion, the relative contribution of $S$ and $A$ to $C$ is a function of their relative levels. Thus, $\Omega_{S}$ and $\Omega_{A}$ are to be defined as a function of $S$ and $A$, respectively. Using a power function for this purpose,

$$
\begin{aligned}
& \Omega_{S}=(\gamma S)^{\beta}=\gamma^{\beta} S^{\beta}, \\
& \Omega_{A}=(\gamma A)^{\beta}=\gamma^{\beta} A^{\beta},
\end{aligned}
$$

where $\gamma>0$ and $\beta \geq 0$. In this formulation, $\gamma$ is an arbitrary scaling constant. For instance, if conflict were expressed on a scale from 0 to 10 instead of a scale from 0 to $1, \gamma$ would be 10 times smaller. In addition, $\beta$ is a conflict-aversion parameter. The greater $\beta$, the greater the aversion to conflict. Substituting Equations A4 and A5 into Equation A3,

$$
C=\frac{A^{\beta}}{S^{\beta}+A^{\beta}} S+\frac{S^{\beta}}{S^{\beta}+A^{\beta}} A .
$$

Thus, $\gamma^{\beta}$ drops out and $C$ can be written as a function of $S, A$, and $\beta$ only. If $\beta=0$, representing an absence of conflict aversion, $C$ is the arithmetic average of $S$ and $A$ :

$$
C=\frac{A^{0}}{S^{0}+A^{0}} S+\frac{S^{0}}{S^{0}+A^{0}} A=1 / 2 S+1 / 2 A=1 / 2(S+A) .
$$

To illustrate this integration rule, $S=.5$ and $A=.5$ yield $C=.5$, and so do $S=.1$ and $A=.9$. Thus, $S$ and $A$ contribute equally to $C$. Alternatively, if $\beta=1 / 2$, representing a specific degree of conflict aversion, $C$ is the geometric average of $S$ and $A$ :

$$
\begin{aligned}
C=\frac{A^{1 / 2}}{S^{1 / 2}+A^{1 / 2}} S+\frac{S^{1 / 2}}{S^{1 / 2}+A^{1 / 2}} A & \\
& =S^{1 / 2} A^{1 / 2} \frac{S^{1 / 2}+A^{1 / 2}}{S^{1 / 2}+A^{1 / 2}}=S^{1 / 2} A^{1 / 2}=(S A)^{1 / 2} .
\end{aligned}
$$

To illustrate this integration rule, $S=.5$ and $A=.5$ yield $C=.5$, but $S=$ .1 and $A=.9$ yield $C=.3$. Thus, $S$, the lower level of conflict, contributes more to $C$. More generally, if we compare Equations A7 and A8, we can verify that $(S A)^{1 / 2}=1 / 2(S+A)$ if and only if $S=A$ and that $(S A)^{1 / 2}<$ $1 / 2(S+A)$ otherwise, as required by conflict aversion.
The conflict generated by each concern is a function of conflict from a preliminary impression, updated by conflict from subsequent deliberation. Again applying Anderson's (1971) integration theory,

$$
\begin{gathered}
S=\left(1-w_{S}\right) S_{0}+w_{S} S_{1}, \\
A=\left(1-w_{A}\right) A_{0}+w_{A} A_{1} .
\end{gathered}
$$

where $0 \leq w_{S}, w_{A}<1$. The updating parameters, $w_{S}$ and $w_{A}$, are relative weights, each of which can be written as a function of an absolute weight:

$$
\begin{aligned}
& w_{S}=\frac{\omega_{S}}{1+\omega_{S}}, \\
& w_{A}=\frac{\omega_{A}}{1+\omega_{A}},
\end{aligned}
$$

where $\omega_{S}, \omega_{A} \geq 0$. The preliminary conflict generated by each concern, $S_{0}$ and $A_{0}$, is a drive for focused deliberation. Therefore, $\omega_{S}$ and $\omega_{A}$ are to be defined as a function of $S_{0}$ and $A_{0}$. Again using a power function for this purpose,

$$
\begin{aligned}
& \omega_{S}=\left(\gamma S_{0}\right)^{\alpha}, \\
& \omega_{A}=\left(\gamma A_{0}\right)^{\alpha},
\end{aligned}
$$

where $\alpha \geq 0$. In this formulation, $\gamma$ is the same arbitrary scaling constant as in Equations A4 and A5. In addition, $\alpha$ is a drive-capacity parameter The greater $\alpha$, the greater the drive capacity of the preliminary conflict.

Finally, the conflict generated by concern about sacrifice and concern about argumentation during deliberation is related in opposite directions to tradeoff size, $T$. Thus, $S_{1}$ and $A_{1}$ are to be defined as functions of $T$. Using linear functions for this purpose,

$$
\begin{gathered}
S_{1}=\delta T, \\
A_{1}=\delta(1-T),
\end{gathered}
$$

where $\delta>0$ and, without loss of generality, tradeoff size is expressed on a scale from 0 to 1 . In this formulation, $\delta$ is an arbitrary constant scaling tradeoff size with respect to conflict. If, as in the present analysis, tradeoff size is expressed on the same scale as conflict, $\delta=1$.

\section{Estimation of Parameters}

We estimate the model from the experimental results displayed in Figure 6 (two of the three conditions: equal and unequal attribute importance) and those displayed in the top panel of Figure 8 (both conditions: need and no need for justification). Each condition provides three data points, corresponding to the three tradeoff sizes (small, intermediate, and large). Thus, we arrive at a total of 12 data points.

The model requires estimation of 11 parameters, viz., $S_{0}$ and $A_{0}$ (the conflict from the preliminary impression) for all four conditions, $\gamma$ (the arbitrary scaling constant), $\alpha$ (the drive-capacity parameter), and $\beta$ (the conflict-aversion parameter). To save one degree of freedom, we estimate the model under the assumption that $\beta=1 / 2$, so that final conflict is given by Equation A8 rather than Equation A6. Testing a new model with only two degrees of freedom is not unprecedented (e.g., Tversky, 1972) nor uninformative (e.g., testing a linear model on three data points forming an isosceles triangle has only one degree of freedom but yields a zero fit).

Equations A13 and A14 were substituted into Equations A11 and A12, respectively, which were substituted into Equations A9 and A10, respectively, which were substituted into Equation A8 to predict final conflict. 
We ran a nonlinear regression, minimizing the sum of squared deviations between observed and predicted conflict, with the use of the Simplex and Quasi-Newton estimation algorithm (StatSoft, 2003). Given the linear functions in Equations A15 and A16, we set $S_{1}$ to 1/6, 3/6, and 5/6, and set $A_{1}$ to $5 / 6,3 / 6$, and $1 / 6$, for small, intermediate, and large tradeoffs, respectively. These specific settings affect the estimates of $\gamma$ and $\alpha$ but not the estimates of $S_{0}$ and $A_{0}$, which are of prime substantive interest.

The fit of the model was nearly perfect, in that $100 \% \times R^{2}=99.92 \%$ of the variance in observed conflict was accounted for by the variance in predicted conflict. The estimates of $\gamma$ and $\alpha$ (standard errors in parentheses) were $\hat{\gamma}=1.85(0.01)$ and $\hat{\gamma}=8.14(0.67)$. Figure A1 displays the estimated relation between the preliminary conflict, denoted $K_{0}$, and the updating parameter, denoted $w_{K}$, where $K$ is either $S$ or $A$ :

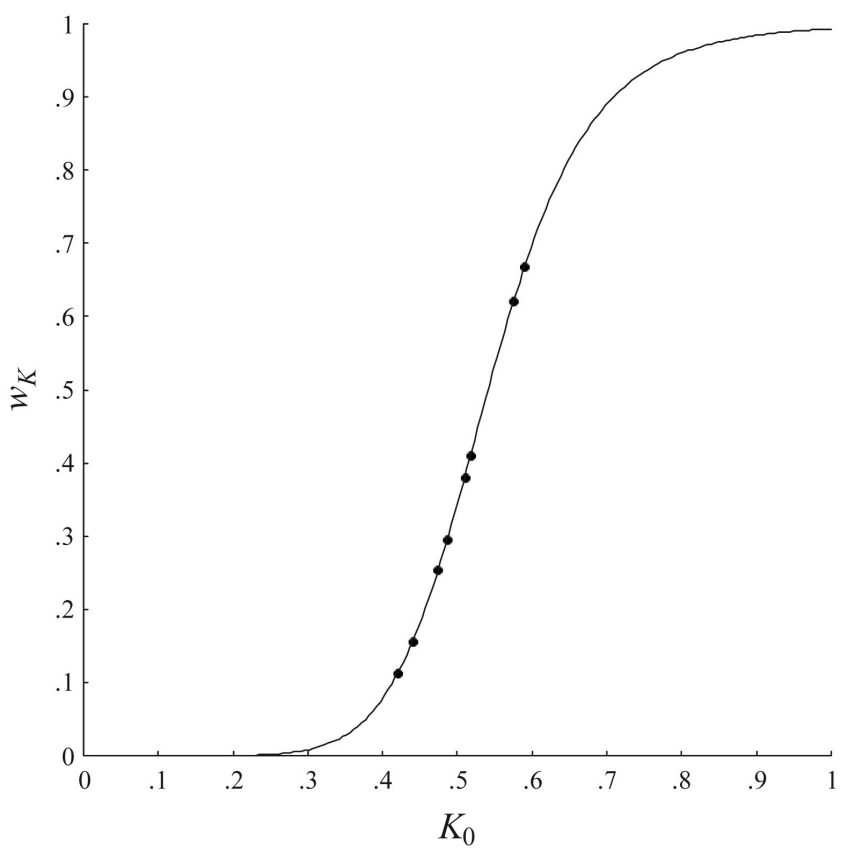

Figure A1. The estimated relation between the preliminary conflict, $K_{0}$, and the updating parameter, $w_{K}$. Filled circles denote equal attribute importance and no need for justification. Filled squared denote unequal attribute importance and no need for justification. Unfilled squared denote unequal attribute importance and need for justification.
Table A1

Estimates of Preliminary Conflict

\begin{tabular}{ccccc} 
& \multicolumn{2}{c}{ Experimental condition } & & \\
\cline { 2 - 4 } Experiment & $\begin{array}{c}\text { Need for } \\
\text { justification }\end{array}$ & $\begin{array}{c}\text { Attribute } \\
\text { importance }\end{array}$ & $\hat{S}_{0}$ & $\hat{A}_{0}$ \\
\hline \multirow{2}{*}{1} & No & Equal & .590 & .575 \\
& & & $(.009)$ & $(.008)$ \\
1 & No & Unequal & .518 & .421 \\
& & & $(.003)$ & $(.002)$ \\
2 & No & Unequal & .509 & .440 \\
& & & $(.003)$ & $(.002)$ \\
2 & Yes & Unequal & .474 & .486 \\
& & & $(.002)$ & $(.002)$ \\
\hline
\end{tabular}

Note. Standard errors are in parentheses.

$$
w_{K}=\frac{\left(\hat{\gamma} K_{0}\right)^{\hat{\alpha}}}{1+\left(\hat{\gamma} K_{0}\right)^{\hat{\alpha}}} \text {. }
$$

The points in Figure A1 refer to the estimates of the preliminary conflict generated by each concern, $\hat{S}_{0}$ and $\hat{A}_{0}$, in each of the four experimental conditions. These estimates, and their standard errors, are provided in Table A1.

\section{Parametric Tests of Hypotheses}

The reasoning underlying $H 1.2$ was that, in comparison to a situation of equal attribute importance, a situation of unequal attribute importance would lead to a decrease in $S_{0}$ as well as a decrease in $A_{0}$ but that the decrease in $A_{0}$ would be greater than the decrease in $S_{0}$. The estimates confirm this pattern. The decrease in $\hat{S}_{0}$ was significant, $.518-.590=$ $-.072, t(2)=-8.94, p=.01$, and so was the decrease in $\hat{A}_{0}, .421-.575=$ $-.154, t(2)=-14.93, p=.00$. Moreover, the decrease in $\hat{A}_{0}$ was significantly greater than the decrease in $\hat{S}_{0},-.154-(-.072)=-.082, t(2)=$ $-16.92, p=.00$.

The reasoning underlying $H 2$ was that, in comparison to a situation without a need for justification, a situation with a need for justification would lead to a decrease in $S_{0}$ but an increase in $A_{0}$. The estimates confirm this pattern. The decrease in $\hat{S}_{0}$ was significant, $.474-.509=-.035, t(2)=$ $-9.18, p=.01$, and so was the increase in $\hat{A}_{0}, .486-.440=.046, t(2)=$ $13.16, p=.00$.

Received May 4, 2005

Revision received January 19, 2006

Accepted January 19, 2006 